ESAIM: COCV 18 (2012) 1097-1121

DOI: $10.1051 / \mathrm{cocv} / 2011191$
ESAIM: Control, Optimisation and Calculus of Variations

www.esaim-cocv.org

\title{
ASYMPTOTIC STABILITY OF STATIONARY SOLUTIONS TO THE DRIFT-DIFFUSION MODEL IN THE WHOLE SPACE
}

\author{
Ryo Kobayashi ${ }^{1,2}$, Masakazu Yamamoto ${ }^{3}$ and Shuichi KaWashima ${ }^{4}$
}

\begin{abstract}
We study the initial value problem for the drift-diffusion model arising in semiconductor device simulation and plasma physics. We show that the corresponding stationary problem in the whole space $\mathbb{R}^{n}$ admits a unique stationary solution in a general situation. Moreover, it is proved that when $n \geq 3$, a unique solution to the initial value problem exists globally in time and converges to the corresponding stationary solution as time tends to infinity, provided that the amplitude of the stationary solution and the initial perturbation are suitably small. Also, we show the sharp decay estimate for the perturbation. The stability proof is based on the time weighted $L^{p}$ energy method.
\end{abstract}

Mathematics Subject Classification. 35K45, 35B35, 82D10, 82D37.

Received September 26, 2011.

Published online 16 January 2012.

\section{INTRODUCTION}

We study the following drift-diffusion model arising in semiconductor device simulation and plasma physics:

$$
\left\{\begin{array}{l}
u_{t}-\Delta u+\nabla \cdot(u \nabla \psi)=0, \\
v_{t}-\Delta v-\nabla \cdot(v \nabla \psi)=0, \\
-\Delta \psi=-(u-v)+g(x),
\end{array}\right.
$$

with the initial conditions

$$
u(x, 0)=u_{0}(x), \quad v(x, 0)=v_{0}(x) .
$$

Here $u=u(x, t)$ and $v=v(x, t)$ denote the electron and the hole densities, respectively, in the semiconductor, while $\psi=\psi(x, t)$ is the electric potential and $g=g(x)$ is the impurity doping profile.

\footnotetext{
Keywords and phrases. Drift-diffusion model, stability, decay estimates, weighted energy method.

1 Graduate School of Mathematics, Kyushu University, 819-0395 Fukuoka, Japan.

2 Information Systems Department, Information \& Communication Devision, Kyushu Electric Power Co. Inc., 810-8720 Fukuoka, Japan.

3 Mathematical Institute, Tohoku University, 980-8578 Sendai, Japan. yamamoto@math.tohoku.ac.jp

4 Faculty of Mathematics, Kyushu University, 819-0395 Fukuoka, Japan. kawashim@math.kyushu-u.ac.jp
} 
The initial value problem (1.1), (1.2) in the whole space $\mathbb{R}^{n}$ was considered by Kurokiba and Ogawa in [12]. They showed the global existence of solutions for nonnegative initial data $\left(u_{0}, v_{0}\right)(x)$ and for $g(x)$ in $L^{p}$ spaces. Asymptotic behavior for $t \rightarrow \infty$ of these global solutions was studied in [1,10] in a special situation where $g(x)=0$. We know that these global solutions decay to zero in $L^{p}$ norm at the rate $t^{-(n / 2)(1-1 / p)}$ as $t \rightarrow \infty$, provided that the initial data are in $L^{1} \cap L^{\infty}$. For the details, we refer the reader to $[1,10]$.

Our drift-diffusion model (1.1) is a parabolic-elliptic system. Similar parabolic-elliptic systems also appear in other models, such as an astrophysical model (a model of gravitating particles) and a model of chemotaxis (see, for example, $[3,5,9,20]$ and references therein). For mathematical theory of those models, we refer the reader to $[2-4,15,17]$ and references therein.

In this paper we study (1.1), (1.2) for $g(x)$ satisfying

$$
g(x) \rightarrow g_{\infty}
$$

as $|x| \rightarrow \infty$, where $g_{\infty}$ is a real constant state. For the initial data, we assume that $u_{0}(x) \rightarrow u_{\infty}$ and $v_{0}(x) \rightarrow v_{\infty}$ as $|x| \rightarrow \infty$, where $u_{\infty}$ and $v_{\infty}$ are nonnegative constants. In order to discuss the asymptotic behavior of solutions to $(1.1),(1.2)$ in this situation, we need to study the corresponding stationary problem in the whole space $\mathbb{R}^{n}$ :

$$
\left\{\begin{array}{l}
-\Delta u+\nabla \cdot(u \nabla \psi)=0, \\
-\Delta v-\nabla \cdot(v \nabla \psi)=0, \\
-\Delta \psi=-(u-v)+g(x),
\end{array}\right.
$$

with the requirements

$$
u(x) \rightarrow u_{\infty}, \quad v(x) \rightarrow v_{\infty}, \quad \psi(x) \rightarrow 0
$$

as $|x| \rightarrow \infty$, where $u_{\infty}$ and $v_{\infty}$ are the above nonnegative constants. It is also assumed that the derivatives of our stationary solutions tend to zero as $|x| \rightarrow \infty$. In this situation we must have

$$
-\left(u_{\infty}-v_{\infty}\right)+g_{\infty}=0 .
$$

The stationary problems for (1.3) in a bounded domain $\Omega$ with natural boundary conditions on $\partial \Omega$ were considered in many papers. It is well known that these stationary problems admit unique solutions in the space $L^{2}(\Omega)$ or $L^{p}(\Omega)$. Moreover, these stationary solutions are asymptotically stable in the sense that the timedependent solutions to the corresponding initial-boundary value problem for (1.1) converge to these stationary solutions exponentially as $t \rightarrow \infty$. See, for example, $[1,14]$.

Our stationary equations (1.3) in the whole space $\mathbb{R}^{n}$ can be reduced to a single equation. This can be verified as follows. We rewrite the first equation (1.3a) as $\nabla \cdot\left(\mathrm{e}^{\psi} \nabla\left(u \mathrm{e}^{-\psi}\right)\right)=0$. We multiply this equation by $u \mathrm{e}^{-\psi}-u_{\infty}$ and integrate over $\mathbb{R}^{n}$. This gives $u \mathrm{e}^{-\psi}=u_{\infty}$. Similarly, we get from (1.3b) that $v \mathrm{e}^{\psi}=v_{\infty}$. Consequently, we have

$$
u=u_{\infty} \mathrm{e}^{\psi}, \quad v=v_{\infty} \mathrm{e}^{-\psi} .
$$

Substituting these relations in (1.3c) and subtracting (1.4), we obtain

$$
-\Delta \psi=-u_{\infty}\left(\mathrm{e}^{\psi}-1\right)-v_{\infty}\left(1-\mathrm{e}^{-\psi}\right)+g(x)-g_{\infty} .
$$

This is the reduced stationary equation in which $u_{\infty}$ and $v_{\infty}$ can be considered as nonnegative parameters of the problem.

In this paper we first show the existence and uniqueness of stationary solutions to (1.6) with $u_{\infty}, v_{\infty}>0$ in the whole space $\mathbb{R}^{n}$. This is an improvement on our previous result obtained in [11] under the restrictions $u_{\infty}=v_{\infty}>0$ and $g_{\infty}=0$. As in [11], our existence proof is based on a fixed point theorem of the LeraySchauder type (called the Browder-Potter fixed point theorem [18]). A crucial point of the proof is to derive the a priori estimate of stationary solutions and this can be done by using the weighted $L^{p}$ energy method. 
The second purpose of this paper is to show the asymptotic stability of the above stationary solution when $n \geq 3$. We prove that a unique solution to the initial value problem (1.1), (1.2) exists globally in time and converges to the corresponding stationary solution as $t \rightarrow \infty$, provided that the amplitude of the stationary solution and the initial perturbation are suitably small. This stability result is based on the $L^{p}$ energy method. Moreover, by employing the time weighted $L^{p}$ energy method (which is a modification of the one used in $[8,10]$ ), we obtain the rate of convergence toward the stationary solution. When the initial perturbation is in $L^{2} \cap L^{q}$ for $q$ with $2 \leq q<n$, the convergence rate obtained in $L^{p}$ norm is $t^{-(n / 2)(1 / 2-1 / p)}$ for $2 \leq p \leq q$ and this rate is just equal to the optimal $L^{p}-L^{2}$ decay rate for the heat equation (see Thm. 4.3). On the other hand, we could not prove such a sharp decay estimate for $n \leq q<\infty$ and $n \leq p \leq q$ (see Thm. 4.5). This provides a striking contrast to the corresponding result for $g(x)=0$ in [10]. Our result may suggest that $p=n$ is the critical exponent in showing the optimal decay in $L^{p}$ for our problem.

This paper consists of five sections. In Section 2, we introduce several inequalities which are used in this paper. We show the existence and uniqueness of stationary solutions in Section 3. In Section 4, we discuss the asymptotic stability of stationary solutions in $L^{p}(2 \leq p<\infty)$. Finally in Section 5 , we consider the decay of the derivative of perturbations in order to derive the asymptotic stability of stationary solutions in $L^{\infty}$.

Notations. For $x=\left(x_{1}, \ldots, x_{n}\right) \in \mathbb{R}^{n}$, we denote by $\partial_{x_{i}}$ the differentiation with respect to $x_{i}$. Also, for a nonnegative integer $k, \partial_{x}^{k}$ denotes the totality of all the $k$-th order differentiations with respect to $x \in \mathbb{R}^{n}$. The symbols $\nabla=\partial_{x}=\left(\partial_{x_{1}}, \ldots, \partial_{x_{n}}\right)$ and $\Delta=\sum_{i=1}^{n} \partial_{x_{i}}^{2}$ denote the gradient and the Laplacian in the $n$-dimensional space, respectively.

For $1 \leq p \leq \infty, L^{p}=L^{p}\left(\mathbb{R}^{n}\right)$ denotes the usual Lebesgue space on $\mathbb{R}^{n}$ with the norm $\|\cdot\|_{L^{p}}$. Let $s$ be a nonnegative integer. Then the corresponding Sobolev space $W^{s, p}=W^{s, p}\left(\mathbb{R}^{n}\right)$ is defined by $W^{s, p}=$ $\left\{f \in L^{p} ; \partial_{x}^{k} f \in L^{p}\right.$ for $\left.k \leq s\right\}$. When $p=2$, we write $H^{s}=W^{s, 2}$. For $\alpha \in \mathbb{R}, L_{\alpha}^{p}=L_{\alpha}^{p}\left(\mathbb{R}^{n}\right)$ denotes the weighted $L^{p}$ space on $\mathbb{R}^{n}$, which consists of functions $f$ satisfying $(1+|x|)^{\alpha} f \in L^{p}$, equipped with the norm $\|f\|_{L_{\alpha}^{p}}=\left\|(1+|x|)^{\alpha} f\right\|_{L^{p}}$. In particular, for $1 \leq p<\infty$, we have

$$
\|f\|_{L_{\alpha}^{p}}=\left(\int_{\mathbb{R}^{n}}(1+|x|)^{\alpha p}|f(x)|^{p} \mathrm{~d} x\right)^{1 / p} .
$$

The corresponding weighted Sobolev space $W_{\alpha}^{s, p}=W_{\alpha}^{s, p}\left(\mathbb{R}^{n}\right)$ is defined as $W_{\alpha}^{s, p}=\left\{f \in L_{\alpha}^{p} ; \partial_{x}^{k} f \in L_{\alpha}^{p}\right.$ for $k \leq s\}$. We write $H_{\alpha}^{s}=W_{\alpha}^{s, 2}$ for $p=2$.

In this paper, various positive constants are denoted by $C$ or $c$ without confusion.

\section{Preliminaries}

In this section we give several preliminary inequalities used in the paper. First, we consider the Poisson equation

$$
-\Delta \psi=f
$$

The corresponding fundamental solution $K(x)$ is given by

$$
K(x)= \begin{cases}\frac{1}{(n-2)\left|S^{n-1}\right|}|x|^{-(n-2)} & (n \geq 3), \\ \frac{1}{2 \pi} \log |x| & (n=2),\end{cases}
$$

where $\left|S^{n-1}\right|=2 \pi^{n / 2} / \Gamma(n / 2)$ is the surface integral of the $(n-1)$-dimensional unit ball. Then the solution to (2.1) is given formally as $\psi(x)=(K * f)(x)$ so that we have formally

$$
\nabla \psi(x)=(\nabla K * f)(x) .
$$


In this paper we always define the gradient of the solution to the Poisson equation (2.1) by the formula (2.2). Then, applying Hardy-Littlewood-Sobolev inequality, we have:

Lemma 2.1 (special Hardy-Littlewood-Sobolev inequality $([19,21])$. Let $n \geq 2,1<r<n$ and $1 / r^{*}=$ $1 / r-1 / n$. If $\psi$ is a solution to the Poisson equation (2.1), then we have

$$
\|\nabla \psi\|_{L^{r^{*}}} \leq C\|f\|_{L^{r}}
$$

Also we have the following elliptic estimate in the weighted space $L_{\alpha}^{p}$.

Lemma $2.2([6,13])$. Let $n \geq 1,1<p<\infty$ and $-n / p<\alpha<n(1-1 / p)$. Then, for $\psi \in L_{l o c}^{1}$ with $\Delta \psi \in L_{\alpha}^{p}$, we have

$$
\left\|\partial_{x}^{2} \psi\right\|_{L_{\alpha}^{p}} \leq C\|\Delta \psi\|_{L_{\alpha}^{p}}
$$

Next, we list up several interpolation inequalities which are frequently used in this paper.

Lemma 2.3 (Gagliardo-Nirenberg inequality [16]). Let $n \geq 1$. Let $1 \leq p, q, r \leq \infty$, and let $k$ be a positive integer. Then for any integer $j$ with $0 \leq j<k$, we have

$$
\left\|\partial_{x}^{j} u\right\|_{L^{p}} \leq C\left\|\partial_{x}^{k} u\right\|_{L^{q}}^{a}\|u\|_{L^{r}}^{1-a}
$$

where

$$
\frac{1}{p}=\frac{j}{n}+a\left(\frac{1}{q}-\frac{k}{n}\right)+(1-a) \frac{1}{r}
$$

for a satisfying $j / k \leq a \leq 1$; there are the following exceptional cases:

(i) if $j=0, q k<n$ and $r=\infty$, then we made the additional assumption that either $u(x) \rightarrow 0$ as $|x| \rightarrow \infty$ or $u \in L^{q^{\prime}}$ for some $0<q^{\prime}<\infty$;

(ii) if $1<r<\infty$, and $k-j-n / r$ is a nonnegative integer, then (2.5) holds only for a satisfying $j / k \leq a<1$.

As a special case of (2.5), we have the following estimate for $n \geq 2$ and $1<r<n$ :

$$
\|u\|_{L^{r^{*}}} \leq C\left\|\partial_{x} u\right\|_{L^{r}},
$$

where $1 / r^{*}=1 / r-1 / n$.

Lemma $2.4([7,8])$. Let $n \geq 1$. Then we have

$$
\|u\|_{L^{p}} \leq C\left\|\nabla\left(|u|^{p / 2}\right)\right\|_{L^{2}}^{2 \gamma /(1+\gamma p)}\|u\|_{L^{q}}^{1 /(1+\gamma p)}
$$

for $2 \leq p<\infty$ and $1 \leq q \leq p$, where $\gamma=(n / 2)(1 / q-1 / p)$.

Lemma 2.5 ([8]). Let $n \geq 1$. Then we have

$$
\|\partial u\|_{L^{p}} \leq C\left\|\partial\left(|\partial u|^{p / 2}\right)\right\|_{L^{2}}^{2 /(p+2)}\|u\|_{L^{p}}^{2 /(p+2)}
$$

for $2 \leq p<\infty$ and

$$
\|\partial u\|_{L^{p}}^{p / 2} \leq C\left\|\partial\left(|\partial u|^{p / 2}\right)\right\|_{L^{2}}^{1-2 / p}\left\|\partial\left(|u|^{p / 2}\right)\right\|_{L^{2}}^{2 / p}
$$

for $p=2$ and $4 \leq p<\infty$, where $\partial=\partial_{x_{i}}$ for $i=1, \ldots, n$. 


\section{Stationary solutions}

We study the stationary equation (1.6), i.e.,

$$
-\Delta \psi=-u_{\infty}\left(\mathrm{e}^{\psi}-1\right)-v_{\infty}\left(1-\mathrm{e}^{-\psi}\right)+\tilde{g}(x)
$$

in the whole space $\mathbb{R}^{n}$, where $\tilde{g}(x)=g(x)-g_{\infty} ; g(x)$ is a given function, and $u_{\infty}, v_{\infty}$ and $g_{\infty}$ are constants satisfying $u_{\infty}, v_{\infty}>0$ and (1.4). It is known from [11] that this equation has a unique solution in the special case where $u_{\infty}=v_{\infty}>0$ and $g_{\infty}=0$. Here we prove the existence and uniqueness of solutions to (3.1) without this restriction. We put

$$
F[\psi]=u_{\infty}\left(\mathrm{e}^{\psi}-1\right)+v_{\infty}\left(1-\mathrm{e}^{-\psi}\right)
$$

and rewrite (3.1) as

$$
-\Delta \psi+F[\psi]=\tilde{g}(x) .
$$

First, similarly to [11], we show the uniqueness of solutions.

Theorem 3.1 (uniqueness). Let $u_{\infty}>0$ and $v_{\infty}>0$. Let $n \geq 1$ and $2 \leq p<\infty$, and suppose that $\tilde{g} \in L^{p}$. Then the solutions to equation (3.1) are unique in the space $W^{1, p} \cap L^{\infty}$.

Proof. Equation (3.3) is rewritten as

$$
-\Delta \psi+a_{1} \psi+G[\psi]=\tilde{g}(x)
$$

where $a_{1}=2 \sqrt{u_{\infty} v_{\infty}}$ and

$$
G[\psi]=F[\psi]-a_{1} \psi=u_{\infty}\left(\mathrm{e}^{\psi}-1\right)+v_{\infty}\left(1-\mathrm{e}^{-\psi}\right)-2 \sqrt{u_{\infty} v_{\infty}} \psi
$$

We notice that $G$ satisfies $(a-b)(G[a]-G[b]) \geq 0$ for all $a, b \in \mathbb{R}$ since the inequality $G^{\prime}[a] \geq 0$ holds. Let $\psi_{1}$ and $\psi_{2}$ be solutions to (3.1). Then the difference $\psi=\psi_{1}-\psi_{2}$ satisfies the equation

$$
-\Delta \psi+a_{1} \psi+\left(G\left[\psi_{1}\right]-G\left[\psi_{2}\right]\right)=0 .
$$

We multiply (3.5) by $|\psi|^{p-2} \psi$ to obtain

$$
a_{1}|\psi|^{p}+c_{0}\left|\nabla\left(|\psi|^{p / 2}\right)\right|^{2}+|\psi|^{p-2}\left(\psi_{1}-\psi_{2}\right)\left(G\left[\psi_{1}\right]-G\left[\psi_{2}\right]\right)-\nabla \cdot\left(|\psi|^{p-2} \psi \nabla \psi\right)=0,
$$

where $c_{0}=4(p-1) / p^{2}$. Integrating over $\mathbb{R}^{n}$ and noting that $\left(\psi_{1}-\psi_{2}\right)\left(G\left[\psi_{1}\right]-G\left[\psi_{2}\right]\right) \geq 0$, we have

$$
\|\psi\|_{L^{p}}^{p}+\left\|\nabla\left(|\psi|^{p / 2}\right)\right\|_{L^{2}}^{2} \leq 0,
$$

which shows that $\psi=0$. This completes the proof.

Next, similarly to [11], we prove the existence of solutions to (3.1) by applying the fixed point theorem of the Leray-Schauder type (called the Browder-Potter fixed point theorem [18]). For lower dimensional case $1 \leq n \leq 3$, we have the following existence theorem in the weighted $L^{2}$ spaces.

Theorem 3.2 (existence). Let $u_{\infty}>0$ and $v_{\infty}>0$. Let $1 \leq n \leq 3$ and $\alpha>0$, and suppose that $\tilde{g} \in L_{\alpha}^{2}$. Then equation (3.1) has a unique solution $\psi \in H_{\alpha}^{2}\left(\subset L_{\alpha}^{\infty}\right)$ such that

$$
\|\psi\|_{H_{\alpha}^{2}} \leq C\|\tilde{g}\|_{L_{\alpha}^{2}}
$$

Moreover, if $\tilde{g} \in H_{\alpha}^{s}$ for an integer $s \geq 1$, then the solution verifies the additional regularity $\psi \in H_{\alpha}^{s+2}$.

For higher dimensional case $n \geq 2$, we have a similar existence theorem in the weighted $L^{p}$ spaces with $n<p<\infty$. 
Theorem 3.3 (existence). Let $u_{\infty}>0$ and $v_{\infty}>0$. Let $n \geq 2, n<p<\infty$ and $\alpha>0$, and suppose that $\tilde{g} \in L_{\alpha}^{p}$. Then equation (3.1) has a unique solution $\psi \in W_{\alpha}^{1, p}\left(\subset L_{\alpha}^{\infty}\right)$ with $\nabla\left(\left|\partial_{x} \psi\right|^{p / 2}\right) \in L_{\alpha p / 2}^{2}$ such that

$$
\|\psi\|_{W_{\alpha}^{1, p}}^{p}+\left\|\nabla\left(\left|\partial_{x} \psi\right|^{p / 2}\right)\right\|_{L_{\alpha p / 2}^{2}}^{2} \leq C\|\tilde{g}\|_{L_{\alpha}^{p}}^{p} .
$$

Moreover, if $\tilde{g} \in W_{\alpha}^{s, p}$ for an integer $s \geq 1$, then the solution verifies the additional regularity $\psi \in W_{\alpha}^{s+1, p}$ with $\nabla\left(\left|\partial_{x}^{s+1} \psi\right|^{p / 2}\right) \in L_{\alpha p / 2}^{2}$.

Remark 3.4. When $\tilde{g} \in L_{\alpha}^{2} \cap L_{\alpha}^{p}$ in the above theorems, the solution $\psi$ is in $H_{\alpha}^{2} \cap W_{\alpha}^{1, p}$ with $\nabla\left(\left|\partial_{x} \psi\right|^{p / 2}\right) \in L_{\alpha p / 2}^{2}$ and satisfies the estimates (3.6) and (3.7). Moreover, we have the regularity $\partial_{x}^{2} \psi \in L_{\alpha^{\prime}}^{p}$, where $0 \leq \alpha^{\prime} \leq \alpha$ and $\alpha^{\prime}<n(1-1 / p)$; this regularity follows from the fact that $\Delta \psi \in L_{\alpha}^{p}$ and the estimate (2.4).

These existence theorems can be proved by the same method employed in [11] where the special case $u_{\infty}=$ $v_{\infty}>0, g_{\infty}=0$ is treated. So we will only give the outline of the proof. As in [11], we use the following fixed point theorem of the Leray-Schauder type.

Theorem 3.5 (Browder-Potter [18]). Let $X$ be a Banach space and let $S$ be a closed convex subset of $X$. Let $\Phi_{\lambda}(\psi)=\Phi(\psi, \lambda)$ be a continuous mapping of $(\psi, \lambda) \in S \times[0,1]$ into a compact subset of $X$, with the following properties:

(i) $\Phi_{0}(\partial S) \subset S$;

(ii) for each $\lambda \in[0,1], \Phi_{\lambda}$ has no fixed point on $\partial S$.

Then the mapping $\Phi_{1}$ has a fixed point in $S$.

Let $X_{p}$ be a Banach space defined by $X_{p}=L_{\alpha / 2}^{p} \cap L_{\alpha / 2}^{\infty}$ with the norm

$$
\|\cdot\|_{X_{p}}=\|\cdot\|_{L_{\alpha / 2}^{p}}+\|\cdot\|_{L_{\alpha / 2}^{\infty}} .
$$

We take $X=X_{2}$ and $X=X_{p}$ with $n<p<\infty$ to prove Theorems 3.2 and 3.3, respectively. We choose a closed convex subset $S$ as $S=\left\{\psi \in X_{p} ;\|\psi\|_{X_{p}} \leq M\right\}$, where $M$ is a suitably large number. We need to define the corresponding mapping to apply the above fixed point theorem. For this purpose, we rewrite (3.1) as

$$
-\Delta \psi+\left(u_{\infty}+v_{\infty}\right) \psi=-H[\psi]+\tilde{g}
$$

where $H[\psi]$ is the nolinear part of $F[\psi]$ in $(3.2)$ and is given by

$$
\begin{aligned}
H[\psi] & =F[\psi]-\left(u_{\infty}+v_{\infty}\right) \psi \\
& =u_{\infty}\left(\mathrm{e}^{\psi}-1\right)+v_{\infty}\left(1-\mathrm{e}^{-\psi}\right)-\left(u_{\infty}+v_{\infty}\right) \psi .
\end{aligned}
$$

We introduce a parameter $\lambda \in[0,1]$ and modify the equation (3.8) as

$$
-\Delta \psi+\left(u_{\infty}+v_{\infty}\right) \psi=\lambda(-H[\psi]+\tilde{g}) .
$$

This equation can be transformed to

$$
\psi=\lambda\left\{-\Delta+\left(u_{\infty}+v_{\infty}\right)\right\}^{-1}(-H[\psi]+\tilde{g}) .
$$

The desired mapping $\Phi_{\lambda}(\psi)$ is then defined as

$$
\Phi_{\lambda}(\psi):=\lambda\left\{-\Delta+\left(u_{\infty}+v_{\infty}\right)\right\}^{-1}(-H[\psi]+\tilde{g}),
$$

so that our solution $\psi$ to equation (3.1) can be obtained as a fixed point of the mapping $\Phi_{1}$, that is, $\psi=\Phi_{1}(\psi)$. 
Let $0 \leq \beta<\alpha$. Then we see that the imbedding $H_{\alpha}^{2} \subset L_{\beta}^{2} \cap L_{\beta}^{\infty}$ is compact for $1 \leq n \leq 3$. Also, the imbedding $W_{\alpha}^{1, p} \subset L_{\beta}^{p} \cap L_{\beta}^{\infty}$ is compact for $n \geq 2$ and $n<p<\infty$. Therefore, as in [11], we can verify that our $\Phi_{\lambda}$ is a continuous mapping of $(\psi, \lambda) \in S \times[0,1]$ into a compact subset of $X$, where $X$ and $S$ are defined above. Also, our $\Phi_{\lambda}$ satisfies the first condition of Theorem 3.5 because of $\Phi_{0}=0$. To check the second condition of Theorem 3.5, we will show the a priori estimate of solutions to (3.10) given in Proposition 3.6 below. Once this is done, our $\Phi_{\lambda}$ satisfies the second condition of Theorem 3.5 for $S$ with suitably large $M$. Consequently, Theorem 3.5 is applicable to our problem and we have a fixed point $\psi \in S$ of the mapping $\Phi_{1}$. This fixed point $\psi$ is a desired stationary solution stated in Theorems 3.2 and 3.3. For the details, we refer the reader to [11].

Therefore, for the proof of Theorems 3.2 and 3.3, it suffices to show the following a priori estimate of solutions to equation (3.10).

Proposition 3.6. Let $u_{\infty}>0, v_{\infty}>0$ and $\lambda \in[0,1]$. Let $n \geq 1,2 \leq p<\infty$ and $\beta \geq 0$, and suppose that $\tilde{g} \in L_{\beta}^{p}$. Let $\psi$ be a solution to the nonlinear equation (3.10) such that $\psi \in L_{\beta / 2}^{p} \cap L_{\beta / 2}^{\infty}$. Then we have $\psi \in W_{\beta}^{1, p}$ and $\nabla\left(\left|\partial_{x} \psi\right|^{p / 2}\right) \in L_{\beta p / 2}^{2}$. Moreover, the solution satisfies the a priori estimate

$$
\|\psi\|_{W_{\beta}^{1, p}}^{p}+\left\|\nabla\left(\left|\partial_{x} \psi\right|^{p / 2}\right)\right\|_{L_{\beta p / 2}^{2}}^{2} \leq C \lambda^{p}\|\tilde{g}\|_{L_{\beta}^{p}}^{p},
$$

where $C$ is a positive constant independent of $\lambda$.

Proof. Let $\tilde{g} \in L_{\beta}^{p}$ and let $\psi \in L_{\beta / 2}^{p} \cap L_{\beta / 2}^{\infty}$ be a solution to the equation (3.10). We have from (3.9) that $|H[\psi]| \leq(1 / 2)\left(u_{\infty}+v_{\infty}\right)|\psi|^{2} \mathrm{e}^{|\psi|}$. Therefore $H[\psi]$ is in $L_{\beta}^{p}$ and satisfies

$$
\|H[\psi]\|_{L_{\beta}^{p}} \leq(1 / 2)\left(u_{\infty}+v_{\infty}\right)\|\psi\|_{L_{\beta / 2}^{\infty}}\|\psi\|_{L_{\beta / 2}^{p}} \mathrm{e}^{\|\psi\|_{L^{\infty}}} .
$$

Consequently, we see that the right hand side of (3.10) belongs to $L_{\beta}^{p}$. Therefore, in the same way as in [11], we conclude that $\psi \in W_{\beta}^{1, p}$ and $\nabla\left(\left|\partial_{x} \psi\right|^{p / 2}\right) \in L_{\beta p / 2}^{2}$.

To prove the a priori estimate (3.11), we employ the weighted $L^{p}$ energy method developed in [11]. The proof is divided into two parts.

Step 1. Let $2 \leq p<\infty$ and $\beta \geq 0$. We first show that

$$
\|\psi\|_{L_{\beta}^{p}}^{p}+\left\|\nabla\left(|\psi|^{p / 2}\right)\right\|_{L_{\beta p / 2}^{2}}^{2} \leq C \lambda^{p}\|\tilde{g}\|_{L_{\beta}^{p}}^{p},
$$

where $C$ is a positive constant independent of $\lambda$. To prove this, we rewrite the equation (3.10) as

$$
-\Delta \psi+a_{\lambda} \psi+\lambda G[\psi]=\lambda \tilde{g}
$$

where

$$
a_{\lambda}=\lambda a_{1}+(1-\lambda) a_{0}, \quad a_{0}=u_{\infty}+v_{\infty}, \quad a_{1}=2 \sqrt{u_{\infty} v_{\infty}},
$$

and $G[\psi]$ is defined in (3.4). Notice that $a_{\lambda} \geq a_{1}>0$. We multiply (3.13) by $|\psi|^{p-2} \psi$ and obtain

$$
c_{0}\left|\nabla\left(|\psi|^{p / 2}\right)\right|^{2}+a_{\lambda}|\psi|^{p}+\lambda|\psi|^{p-2} \psi G[\psi]-\nabla \cdot\left(|\psi|^{p-2} \psi \nabla \psi\right)=\lambda|\psi|^{p-2} \psi \tilde{g},
$$

where $c_{0}=4(p-1) / p^{2}$. Furthermore, multiplying by $(1+|x|)^{\beta p}$, we have

$$
\begin{aligned}
& c_{0}(1+|x|)^{\beta p}\left|\nabla\left(|\psi|^{p / 2}\right)\right|^{2}+a_{\lambda}(1+|x|)^{\beta p}\left(|\psi|^{p}+\lambda|\psi|^{p-2} \psi G[\psi]\right) \\
& -\nabla \cdot\left((1+|x|)^{\beta p}|\psi|^{p-2} \psi \nabla \psi\right)+\beta p(1+|x|)^{\beta p-1}|x|^{-1} x \cdot|\psi|^{p-2} \psi \nabla \psi=\lambda(1+|x|)^{\beta p}|\psi|^{p-2} \psi \tilde{g} .
\end{aligned}
$$

Integrating this equality over $\mathbb{R}^{n}$ and using $\psi G[\psi] \geq 0$, we have

$$
a_{\lambda}\|\psi\|_{L_{\beta}^{p}}^{p}+c_{0}\left\|\nabla\left(|\psi|^{p / 2}\right)\right\|_{L_{\beta p / 2}^{2}}^{2} \leq A_{1}+A_{2},
$$


where

$$
A_{1}=\beta p \int_{\mathbb{R}^{n}}(1+|x|)^{\beta p-1}|\psi|^{p-1}|\nabla \psi| \mathrm{d} x, \quad A_{2}=\lambda \int_{\mathbb{R}^{n}}(1+|x|)^{\beta p}|\psi|^{p-1}|\tilde{g}| \mathrm{d} x .
$$

We estimate the right hand side of (3.14). For the term $A_{1}$, we have

$$
\begin{aligned}
A_{1} & =(\beta / 2) \int_{\mathbb{R}^{n}}(1+|x|)^{\beta p-1}|\psi|^{p / 2}\left|\nabla\left(|\psi|^{p / 2}\right)\right| \mathrm{d} x \\
& \leq(\beta / 2)\|\psi\|_{L_{\beta}^{p}}^{p / 2}\left\|\nabla\left(|\psi|^{p / 2}\right)\right\|_{L_{\beta p / 2-1}^{2}} \leq \varepsilon\|\psi\|_{L_{\beta}^{p}}^{p}+\beta^{2} C_{\varepsilon}\left\|\nabla\left(|\psi|^{p / 2}\right)\right\|_{L_{\beta p / 2-1}^{2}}^{2}
\end{aligned}
$$

for any $\varepsilon>0$, where $C_{\varepsilon}$ is a positive constant depending on $\varepsilon$ but not on $\beta$. Similarly, we can estimate the term $A_{2}$ as

$$
A_{2} \leq \lambda\|\psi\|_{L_{\beta}^{p}}^{p-1}\|\tilde{g}\|_{L_{\beta}^{p}} \leq \varepsilon\|\psi\|_{L_{\beta}^{p}}^{p}+C_{\varepsilon} \lambda^{p}\|\tilde{g}\|_{L_{\beta}^{p}}^{p}
$$

for any $\varepsilon>0$, where $C_{\varepsilon}$ is a positive constant depending on $\varepsilon$ but not on $\beta$. Substituting these estimates in (3.14) and taking $\varepsilon>0$ suitably small, we obtain

$$
\|\psi\|_{L_{\beta}^{p}}^{p}+\left\|\nabla\left(|\psi|^{p / 2}\right)\right\|_{L_{\beta p / 2}^{2}}^{2} \leq C \lambda^{p}\|\tilde{g}\|_{L_{\beta}^{p}}^{p}+\beta^{2} C\left\|\nabla\left(|\psi|^{p / 2}\right)\right\|_{L_{\beta p / 2-1}^{2}}^{2} .
$$

This proves (3.12) for $\beta=0$. Also, (3.16) together with (3.12) for $\beta=0$ gives (3.12) for $0<\beta p \leq 2$. Repeating this procedure, we conclude that (3.12) holds true for any $\beta \geq 0$.

Step 2. Next we show that

$$
\left\|\partial_{x} \psi\right\|_{L_{\beta}^{p}}^{p}+\left\|\nabla\left(\left|\partial_{x} \psi\right|^{p / 2}\right)\right\|_{L_{\beta p / 2}^{2}}^{2} \leq C \lambda^{p}\|\tilde{g}\|_{L_{\beta}^{p}}^{p},
$$

where $C$ is a positive constant independent of $\lambda$. To prove this, we differentiate (3.13) with respect to $x_{i}$ $(i=1, \ldots, n)$, obtaining

$$
-\Delta \psi_{i}+a_{\lambda} \psi_{i}+\lambda \partial_{x_{i}} G[\psi]=\lambda \partial_{x_{i}} \tilde{g}
$$

where we put $\psi_{i}=\partial_{x_{i}} \psi$. We multiply (3.18) by $\left|\psi_{i}\right|^{p-2} \psi_{i}$ to get

$$
\begin{aligned}
& c_{0}\left|\nabla\left(\left|\psi_{i}\right|^{p / 2}\right)\right|^{2}+a_{\lambda}\left|\psi_{i}\right|^{p}+\lambda\left|\psi_{i}\right|^{p-2} \psi_{i} \partial_{x_{i}} G[\psi] \\
& -\nabla \cdot\left(\left|\psi_{i}\right|^{p-2} \psi_{i} \nabla \psi_{i}\right)=\lambda \partial_{x_{i}}\left(\left|\psi_{i}\right|^{p-2} \psi_{i} \tilde{g}\right)-\lambda(p-1)\left|\psi_{i}\right|^{p-2} \partial_{x_{i}} \psi_{i} \tilde{g},
\end{aligned}
$$

where $c_{0}=4(p-1) / p^{2}$. Furthermore, multiplying by $(1+|x|)^{\beta p}$, we have

$$
\begin{aligned}
& c_{0}(1+|x|)^{\beta p}\left|\nabla\left(\left|\psi_{i}\right|^{p / 2}\right)\right|^{2}+(1+|x|)^{\beta p}\left(a_{\lambda}\left|\psi_{i}\right|^{p}+\lambda\left|\psi_{i}\right|^{p-2} \psi_{i} \partial_{x_{i}} G[\psi]\right) \\
& -\nabla \cdot\left((1+|x|)^{\beta p}\left|\psi_{i}\right|^{p-2} \psi_{i} \nabla \psi_{i}\right)+\beta p(1+|x|)^{\beta p-1}|x|^{-1} x \cdot\left|\psi_{i}\right|^{p-2} \psi_{i} \nabla \psi_{i} \\
& =\lambda \partial_{x_{i}}\left((1+|x|)^{\beta p}\left|\psi_{i}\right|^{p-2} \psi_{i} \tilde{g}\right)-\lambda \beta p(1+|x|)^{\beta p-1}|x|^{-1} x_{i}\left|\psi_{i}\right|^{p-2} \psi_{i} \tilde{g}-\lambda(p-1)(1+|x|)^{\beta p}\left|\psi_{i}\right|^{p-2} \partial_{x_{i}} \psi_{i} \tilde{g} .
\end{aligned}
$$

Integrating this equality over $\mathbb{R}^{n}$ and noting that $\psi_{i} \partial_{x_{i}} G[\psi]=G^{\prime}[\psi]\left|\psi_{i}\right|^{2} \geq 0$, we obtain

$$
a_{\lambda}\left\|\psi_{i}\right\|_{L_{\beta}^{p}}^{p}+c_{0}\left\|\nabla\left(\left|\psi_{i}\right|^{p / 2}\right)\right\|_{L_{\beta p / 2}^{2}}^{2} \leq B_{1}+B_{2}+B_{3}
$$

where

$$
\begin{aligned}
& B_{1}=\beta p \int_{\mathbb{R}^{n}}(1+|x|)^{\beta p-1}\left|\psi_{i}\right|^{p-1}\left|\nabla \psi_{i}\right| \mathrm{d} x, \\
& B_{2}=\lambda \beta p \int_{\mathbb{R}^{n}}(1+|x|)^{\beta p-1}\left|\psi_{i}\right|^{p-1}|\tilde{g}| \mathrm{d} x, \\
& B_{3}=\lambda(p-1) \int_{\mathbb{R}^{n}}(1+|x|)^{\beta p}\left|\psi_{i}\right|^{p-2}\left|\partial_{x_{i}} \psi_{i}\right||\tilde{g}| \mathrm{d} x .
\end{aligned}
$$


We estimate the right hand side of (3.19). The term $B_{1}$ is just the same as $A_{1}$ in (3.15), so that we have

$$
B_{1} \leq \varepsilon\left\|\psi_{i}\right\|_{L_{\beta}^{p}}^{p}+\beta^{2} C_{\varepsilon}\left\|\nabla\left(\left|\psi_{i}\right|^{p / 2}\right)\right\|_{L_{\beta p / 2-1}^{2}}^{2}
$$

for any $\varepsilon>0$, where $C_{\varepsilon}$ is a positive constant depending on $\varepsilon$ but not on $\beta$. For the term $B_{2}$, we have

$$
B_{2} \leq \lambda \beta p\left\|\psi_{i}\right\|_{L_{\beta}^{p}}^{p-1}\|\tilde{g}\|_{L_{\beta-1}^{p}} \leq \varepsilon\left\|\psi_{i}\right\|_{L_{\beta}^{p}}^{p}+C_{\varepsilon} \lambda^{p} \beta^{p}\|\tilde{g}\|_{L_{\beta-1}^{p}}^{p}
$$

for any $\varepsilon>0$, where $C_{\varepsilon}$ is a positive constant depending on $\varepsilon$ but not on $\beta$. On the other hand, we can estimate the term $B_{3}$ as

$$
\begin{aligned}
B_{3} & =\lambda(2 / p)(p-1) \int_{\mathbb{R}^{n}}(1+|x|)^{\beta p}\left|\psi_{i}\right|^{p / 2-1}\left|\partial_{x_{i}}\left(\left|\psi_{i}\right|^{p / 2}\right)\right||\tilde{g}| \mathrm{d} x \\
& \leq \lambda(2 / p)(p-1)\left\|\psi_{i}\right\|_{L_{\beta}^{p}}^{p / 2-1}\left\|\partial_{x_{i}}\left(\left|\psi_{i}\right|^{p / 2}\right)\right\|_{L_{\beta p / 2}^{2}}\|\tilde{g}\|_{L_{\beta}^{p}}^{p} \\
& \leq \varepsilon\left(\left\|\psi_{i}\right\|_{L_{\beta}^{p}}^{p}+\left\|\nabla\left(\left|\psi_{i}\right|^{p / 2}\right)\right\|_{L_{\beta p / 2}^{2}}^{2}\right)+C_{\varepsilon} \lambda^{p}\|\tilde{g}\|_{L_{\beta}^{p}}^{p}
\end{aligned}
$$

for any $\varepsilon>0$, where $C_{\varepsilon}$ is a positive constant depending on $\varepsilon$ but not on $\beta$. Here we have used the Hölder inequality with $(p-2) / 2 p+1 / 2+1 / p=1$. Substituting all these estimates in (3.19) and taking $\varepsilon>0$ suitably small, we obtain

$$
\left\|\partial_{x} \psi\right\|_{L_{\beta}^{p}}^{p}+\left\|\nabla\left(\left|\partial_{x} \psi\right|^{p / 2}\right)\right\|_{L_{\beta p / 2}^{2}}^{2} \leq C \lambda^{p}\left(1+\beta^{p}\right)\|\tilde{g}\|_{L_{\beta}^{p}}^{p}+\beta^{2} C\left\|\nabla\left(\left|\partial_{x} \psi\right|^{p / 2}\right)\right\|_{L_{\beta p / 2-1}^{2}}^{2} .
$$

This gives (3.17) for $\beta=0$ and hence for any $\beta \geq 0$.

Now, the desired estimate (3.11) follows from (3.12) and (3.17), and therefore the proof of Proposition 3.6 is complete.

\section{Asymptotic Stability}

In this section we discuss the asymptotic stability of stationary solutions to the drift-diffusion model in $\mathbb{R}^{n}$ with $n \geq 3$. We denote by $(\bar{u}, \bar{v}, \bar{\psi})(x)$ the stationary solution constructed in Theorems 3.2 and 3.3 for $\tilde{g} \in L_{\alpha}^{2} \cap L_{\alpha}^{q_{0}}$ with $q_{0}>n$ and $\alpha>0$. This stationary solution satisfies the estimates

$$
\begin{array}{ll}
\left\|\left(\bar{u}-u_{\infty}, \bar{v}-v_{\infty}, \bar{\psi}\right)\right\|_{W^{1, r}} \leq C\|\tilde{g}\| & \text { for } \quad 2 \leq r \leq \infty, \\
\left\|\left(\bar{u}-u_{\infty}, \bar{v}-v_{\infty}, \bar{\psi}\right)\right\|_{W^{2, p}} \leq C\|\tilde{g}\| & \text { for } \quad 2 \leq p \leq q_{0},
\end{array}
$$

provided that

$$
\|\tilde{g}\|:=\|\tilde{g}\|_{L^{2}}+\|\tilde{g}\|_{L^{q_{0}}}
$$

is bounded by a constant $C$. In fact, it follows from (3.6) and (3.7) that $\|\bar{\psi}\|_{W^{1, p}} \leq C\|\tilde{g}\|$ for $2 \leq p \leq q_{0}$. Since $q_{0}>n$, this together with (2.5) shows that $\|\bar{\psi}\|_{L^{\infty}} \leq C\|\tilde{g}\|$. Therefore, by virtue of equation (3.1), we know that $\|\Delta \bar{\psi}\|_{L^{p}} \leq C\|\tilde{g}\|$ and hence $\left\|\partial_{x}^{2} \bar{\psi}\right\|_{L^{p}} \leq C\|\tilde{g}\|$ for $2 \leq p \leq q_{0}$ by (2.4) (see also [19]). Consequently, we have $\|\bar{\psi}\|_{W^{2, p}} \leq C\|\tilde{g}\|$ for $2 \leq p \leq q_{0}$, which together with (1.5) shows that $\left\|\left(\bar{u}-u_{\infty}, \bar{v}-v_{\infty}\right)\right\|_{W^{2, p}} \leq C\|\tilde{g}\|$ for $2 \leq p \leq q_{0}$. Thus we have proved the second estimate in (4.1). Also, this combined with (2.5) yields the first estimate in (4.1).

Now we look for solutions to the nonstationary problem $(1.1),(1.2)$ in the form

$$
(u, v, \psi)=(\bar{u}, \bar{v}, \bar{\psi})(x)+(w, z, \phi)(x, t)
$$


where $(\bar{u}, \bar{v}, \bar{\psi})$ is the above stationary solution and $(w, z, \phi)$ denotes the corresponding perturbation. The problem is then reduced to

$$
\begin{gathered}
\left\{\begin{array}{l}
w_{t}-\Delta w+u_{\infty} \Delta \phi=-\nabla \cdot f_{1}-\nabla \cdot(w \nabla \phi), \\
z_{t}-\Delta z-v_{\infty} \Delta \phi=\nabla \cdot f_{2}+\nabla \cdot(z \nabla \phi), \\
-\Delta \phi=-(w-z),
\end{array}\right. \\
w(x, 0)=w_{0}(x), \quad z(x, 0)=z_{0}(x),
\end{gathered}
$$

where $w_{0}=u_{0}-\bar{u}, z_{0}=v_{0}-\bar{v}$ and

$$
f_{1}=w \nabla \bar{\psi}+\left(\bar{u}-u_{\infty}\right) \nabla \phi, \quad f_{2}=z \nabla \bar{\psi}+\left(\bar{v}-v_{\infty}\right) \nabla \phi .
$$

First we show the global existence and uniform $L^{p}$ estimate of solutions to the problem (4.3), (4.4).

Theorem 4.1 (global existence). Let $n \geq 3$ and $q_{1} \leq q<\infty$, where $q_{1}=\max \{2, n / 2\}$. Let $(\bar{u}, \bar{v}, \bar{\psi})$ be the stationary solution satisfying (4.1). Suppose that the initial data $\left(w_{0}, z_{0}\right)$ are in $L^{2} \cap L^{q}$ and put $E_{0}=$ $\left\|\left(w_{0}, z_{0}\right)\right\|_{L^{2}}+\left\|\left(w_{0}, z_{0}\right)\right\|_{L^{q}}$. If $E_{0}+\|\tilde{g}\|$ is suitably small $(\|\tilde{g}\|$ is defined in (4.2)), then the problem (4.3), (4.4) has a unique global solution $(w, z, \phi)$ which satisfies the following uniform $L^{p}$ estimate:

$$
\|(w, z)(t)\|_{L^{p}}^{p}+\int_{0}^{t}\left\|\nabla\left(|(w, z)|^{p / 2}\right)(\tau)\right\|_{L^{2}}^{2}+\|(w-z)(\tau)\|_{L^{p}}^{p} \mathrm{~d} \tau \leq C E_{0}^{p}
$$

for $p=2$ and $q_{1} \leq p \leq q$. In particular, we have $\|(w, z)(t)\|_{L^{p}} \leq C E_{0}$ for each $p$ with $2 \leq p \leq q$.

The local existence of solutions to the problem (4.3), (4.4) can be proved by the standard method (cf. [12]). Therefore, for the proof of Theorem 4.1, it suffices to show the a priori estimate of solutions to the problem (4.3), (4.4). We use the following notations.

$$
\begin{aligned}
& E_{p}(t)=\sup _{0 \leq \tau \leq t}\|(w, z)(\tau)\|_{L^{p}}, \\
& D_{p}(t)^{p}=\int_{0}^{t}\left\|\nabla\left(|(w, z)|^{p / 2}\right)(\tau)\right\|_{L^{2}}^{2}+\|(w-z)(\tau)\|_{L^{p}}^{p} \mathrm{~d} \tau,
\end{aligned}
$$

where $2 \leq p<\infty$. Our a priori estimate is then given as follows.

Proposition 4.2 (a priori $L^{p}$ estimate). Let $n \geq 3$ and $q_{1} \leq q<\infty$, where $q_{1}$ is the same as in Theorem 4.1. Let $(\bar{u}, \bar{v}, \bar{\psi})$ be the stationary solution satisfying (4.1) and let $(w, z, \phi)$ be a solution to the problem (4.3), (4.4) corresponding to the initial data $\left(w_{0}, z_{0}\right) \in L^{2} \cap L^{q}$. If $E_{0}+\|\tilde{g}\|$ in Theorem 4.1 is suitably small, then the solution $(w, z, \phi)$ satisfies the following a priori $L^{p}$ estimate:

$$
\left(E_{p}+D_{p}\right)(t) \leq C E_{0}
$$

for $p=2$ and $q_{1} \leq p \leq q$.

Proof. Let $n \geq 3$. The proof is based on the $L^{p}$ energy method employed in [8]. We divide the proof into four parts.

Step 1. Let $2 \leq p \leq q$. We derive the $L^{p}$ energy inequality for the problem (4.3), (4.4). We multiply (4.3a) by $|w|^{p-2} w$. A straightforward computation gives

$$
\begin{aligned}
& \frac{1}{p}\left(|w|^{p}\right)_{t}+c_{0}\left|\nabla\left(|w|^{p / 2}\right)\right|^{2}+u_{\infty}|w|^{p-2} w(w-z)-\nabla \cdot\left(|w|^{p-2} w \nabla w\right) \\
& =-\nabla \cdot\left(|w|^{p-2} w f_{1}+|w|^{p} \nabla \phi\right)+(p-1)\left(|w|^{p-2} \nabla w \cdot f_{1}+|w|^{p-2} w \nabla w \cdot \nabla \phi\right),
\end{aligned}
$$



where $c_{0}=4(p-1) / p^{2}$. Here we have used (4.3c). We integrate (4.8) over $\mathbb{R}^{n}$ to obtain

$$
\frac{1}{p} \frac{\mathrm{d}}{\mathrm{d} t}\|w\|_{L^{p}}^{p}+c_{0}\left\|\nabla\left(|w|^{p / 2}\right)\right\|_{L^{2}}^{2}+u_{\infty} \int_{\mathbb{R}^{n}}|w|^{p-2} w(w-z) \mathrm{d} x \leq(p-1)(I+J),
$$

where we put

$$
I=\int_{\mathbb{R}^{n}}|w|^{p-2}|\nabla w|\left|f_{1}\right| \mathrm{d} x, \quad J=\int_{\mathbb{R}^{n}}|w|^{p-1}|\nabla w||\nabla \phi| \mathrm{d} x .
$$

It follows from (4.5) that $I \leq I_{1}+I_{2}$ with

$$
I_{1}=\int_{\mathbb{R}^{n}}|\nabla \bar{\psi}||w|^{p-1}|\nabla w| \mathrm{d} x, \quad I_{2}=\int_{\mathbb{R}^{n}}\left|\bar{u}-u_{\infty}\right||w|^{p-2}|\nabla w||\nabla \phi| \mathrm{d} x .
$$

We integrate (4.9) with respect to $t$. This yields

$$
\|w(t)\|_{L^{p}}^{p}+\int_{0}^{t}\left\|\nabla\left(|w|^{p / 2}\right)(\tau)\right\|_{L^{2}}^{2} \mathrm{~d} \tau+\int_{0}^{t} \int_{\mathbb{R}^{n}}|w|^{p-2} w(w-z)(\tau) \mathrm{d} x \mathrm{~d} \tau \leq C\left\|w_{0}\right\|_{L^{p}}^{p}+C \int_{0}^{t}(I+J)(\tau) \mathrm{d} \tau,
$$

where we have used $u_{\infty}>0$. We have a similar energy inequality also for $z$.

Step 2. Let $q_{1} \leq p \leq q$. We show the following $L^{p}$ energy inequality:

$$
\left(E_{p}+D_{p}\right)(t)^{p} \leq C E_{0}^{p}+C\|\tilde{g}\|\left(E_{2}+D_{2}+D_{p}\right)(t)^{p}+C\left(E_{p}+D_{2}+D_{p}\right)(t)^{p+1} .
$$

We use (4.12). First, applying the Hölder inequality with $1 / n+1 / 2^{*}+1 / 2=1$ (where $1 / 2^{*}=1 / 2-1 / n$ ) and using (2.6), we estimate the term $I_{1}$ as

$$
\begin{aligned}
I_{1} & =(2 / p) \int_{\mathbb{R}^{n}}|\nabla \bar{\psi}||w|^{p / 2}\left|\nabla\left(|w|^{p / 2}\right)\right| \mathrm{d} x \\
& \leq C\|\nabla \bar{\psi}\|_{L^{n}}\left\|\left(|w|^{p / 2}\right)\right\|_{L^{2^{*}}}\left\|\nabla\left(|w|^{p / 2}\right)\right\|_{L^{2}} \leq C\|\tilde{g}\|\left\|\nabla\left(|w|^{p / 2}\right)\right\|_{L^{2}}^{2}
\end{aligned}
$$

Thus we obtain

$$
\int_{0}^{t} I_{1}(\tau) \mathrm{d} \tau \leq C\|\tilde{g}\| \int_{0}^{t}\left\|\nabla\left(|w|^{p / 2}\right)(\tau)\right\|_{L^{2}}^{2} \mathrm{~d} \tau \leq C\|\tilde{g}\| D_{p}(t)^{p}
$$

Next we estimate the term $I_{2}$. We determine $r$ with $1<r<n$ and $2<r<p$ such that

$$
\frac{1}{r}=\frac{\theta}{2}+\frac{1-\theta}{p}, \quad \theta=\frac{2}{n} .
$$

For this choice of $r$, we see that $1 / n+(1-2 / p) / 2^{*}+1 / 2+1 / r^{*}=1$, where $1 / 2^{*}=1 / 2-1 / n$ and $1 / r^{*}=1 / r-1 / n$. Applying the Hölder inequality with this relation and using (2.6) and (2.3), we have

$$
\begin{aligned}
I_{2} & =(2 / p) \int_{\mathbb{R}^{n}}\left|\bar{u}-u_{\infty}\right||w|^{(p / 2)(1-2 / p)}\left|\nabla\left(|w|^{p / 2}\right)\right||\nabla \phi| \mathrm{d} x \\
& \leq C\left\|\bar{u}-u_{\infty}\right\|_{L^{n}}\left\|\left(|w|^{p / 2}\right)\right\|_{L^{2^{*}}}^{1-2 / p}\left\|\nabla\left(|w|^{p / 2}\right)\right\|_{L^{2}}\|\nabla \phi\|_{L^{r^{*}}} \\
& \leq C\|\tilde{g}\|\left\|\nabla\left(|w|^{p / 2}\right)\right\|_{L^{2}}^{2(1-1 / p)}\|w-z\|_{L^{r}} \\
& \leq C\|\tilde{g}\|\left\|\nabla\left(|w|^{p / 2}\right)\right\|_{L^{2}}^{2(1-1 / p)}\|w-z\|_{L^{2}}^{\theta}\|w-z\|_{L^{p}}^{1-\theta},
\end{aligned}
$$


where we have used the interpolation in $L^{r}$ with the relation (4.15). Consequently, we obtain

$$
\begin{aligned}
\int_{0}^{t} I_{2}(\tau) \mathrm{d} \tau & \leq C\|\tilde{g}\| E_{2}(t)^{\theta(1-2 / p)} \int_{0}^{t}\|w-z\|_{L^{2}}^{2 \theta / p}\left\|\nabla\left(|w|^{p / 2}\right)\right\|_{L^{2}}^{2(1-1 / p)}\|w-z\|_{L^{p}}^{1-\theta} \mathrm{d} \tau \\
& \leq C\|\tilde{g}\| E_{2}(t)^{\theta(1-2 / p)} D_{2}(t)^{2 \theta / p} D_{p}(t)^{(p-1)+(1-\theta)} \leq C\|\tilde{g}\|\left(E_{2}+D_{2}+D_{p}\right)(t)^{p},
\end{aligned}
$$

where we used the Hölder inequality with $\theta / p+(1-1 / p)+(1-\theta) / p=1$.

Finally, we estimate the term $J$. To this end, we choose $r$ and $\lambda$ with $1<r<n, 2 \leq r \leq p$ and $0 \leq \lambda<1$ such that

$$
\frac{\lambda}{2}+\frac{1-\lambda}{2^{*}}+\frac{1}{2}+\frac{1}{r^{*}}=1, \quad \frac{1}{r}=\frac{\lambda}{2}+\frac{1-\lambda}{p},
$$

where $1 / 2^{*}=1 / 2-1 / n$ and $1 / r^{*}=1 / r-1 / n$. This choice is possible because we have $1 / r=2 / n-\lambda / n$ and $\lambda=2(2 p-n) /((n+2) p-2 n)<1$; here we used the restriction $p \geq n / 2$. Then, applying the Hölder inequality with the first relation in (4.17) and using (2.6) and (2.3), we have

$$
\begin{aligned}
J & =(2 / p) \int_{\mathbb{R}^{n}}|w|^{\lambda p / 2}|w|^{(1-\lambda) p / 2}\left|\nabla\left(|w|^{p / 2}\right)\right||\nabla \phi| \mathrm{d} x \\
& \leq C\left\|\left(|w|^{p / 2}\right)\right\|_{L^{2}}^{\lambda}\left\|\left(|w|^{p / 2}\right)\right\|_{L^{2^{*}}}^{1-\lambda}\left\|\nabla\left(|w|^{p / 2}\right)\right\|_{L^{2}}\|\nabla \phi\|_{L^{r^{*}}} \\
& \leq C\|w\|_{L^{p}}^{\lambda p / 2}\left\|\nabla\left(|w|^{p / 2}\right)\right\|_{L^{2}}^{2-\lambda}\|w-z\|_{L^{r}} \\
& \leq C\|w\|_{L^{p}}^{\lambda / 2}\left\|\nabla\left(|w|^{p / 2}\right)\right\|_{L^{2}}^{2-\lambda}\|w-z\|_{L^{2}}^{\lambda}\|w-z\|_{L^{p}}^{1-\lambda},
\end{aligned}
$$

where we have used the interpolation in $L^{r}$ with the second relation in (4.17). Consequently, we obtain

$$
\begin{aligned}
\int_{0}^{t} J(\tau) \mathrm{d} \tau & \leq C E_{p}(t)^{\lambda p / 2+(1-\lambda)} \int_{0}^{t}\left\|\nabla\left(|w|^{p / 2}\right)\right\|_{L^{2}}^{2-\lambda}\|w-z\|_{L^{2}}^{\lambda} \mathrm{d} \tau \\
& \leq C E_{p}(t)^{\lambda p / 2+(1-\lambda)} D_{p}(t)^{(2-\lambda) p / 2} D_{2}(t)^{\lambda} \leq C\left(E_{p}+D_{2}+D_{p}\right)(t)^{p+1},
\end{aligned}
$$

where we used the Hölder inequality with $(2-\lambda) / 2+\lambda / 2=1$.

Now, substituting all these estimates into (4.12), we arrive at

$$
\begin{aligned}
& \|w(t)\|_{L^{p}}^{p}+\int_{0}^{t}\left\|\nabla\left(|w|^{p / 2}\right)(\tau)\right\|_{L^{2}}^{2} \mathrm{~d} \tau+\int_{0}^{t} \int_{\mathbb{R}^{n}}|w|^{p-2} w(w-z)(\tau) \mathrm{d} x \mathrm{~d} \tau \\
& \leq C E_{0}^{p}+C\|\tilde{g}\|\left(E_{2}+D_{2}+D_{p}\right)(t)^{p}+C\left(E_{p}+D_{2}+D_{p}\right)(t)^{p+1} .
\end{aligned}
$$

Similarly, for $z$, we have

$$
\begin{aligned}
& \|z(t)\|_{L^{p}}^{p}+\int_{0}^{t}\left\|\nabla\left(|z|^{p / 2}\right)(\tau)\right\|_{L^{2}}^{2} \mathrm{~d} \tau-\int_{0}^{t} \int_{\mathbb{R}^{n}}|z|^{p-2} z(w-z)(\tau) \mathrm{d} x \mathrm{~d} \tau \\
& \leq C E_{0}^{p}+C\|\tilde{g}\|\left(E_{2}+D_{2}+D_{p}\right)(t)^{p}+C\left(E_{p}+D_{2}+D_{p}\right)(t)^{p+1} .
\end{aligned}
$$

Adding these two inequalities and using the fact that $|w-z|^{p} \leq 2^{p-2}\left(|w|^{p-2} w-|z|^{p-2} z\right)(w-z)$, we obtain the desired estimate (4.13).

Step 3. We show the following $L^{2}$ energy inequality:

$$
\left(E_{2}+D_{2}\right)(t)^{2} \leq C E_{0}^{2}+C\|\tilde{g}\| D_{2}(t)^{2}+C\left(E_{2}+E_{p}+D_{2}\right)(t)^{3},
$$

where $q_{1} \leq p \leq q$. We use (4.12) with $p=2$ :

$$
\|w(t)\|_{L^{2}}^{2}+\int_{0}^{t}\|\nabla w(\tau)\|_{L^{2}}^{2} \mathrm{~d} \tau+\int_{0}^{t} \int_{\mathbb{R}^{n}} w(w-z)(\tau) \mathrm{d} x \mathrm{~d} \tau \leq C\left\|w_{0}\right\|_{L^{2}}^{2}+C \int_{0}^{t}(I+J)(\tau) \mathrm{d} \tau,
$$


where $I$ and $J$ are defined by (4.10) with $p=2$. The term $I_{1}$ in (4.11) with $p=2$ is estimated just in the same way as before. In fact, we have $I_{1} \leq C\|\tilde{g}\|\|\nabla w\|_{L^{2}}^{2}$ and

$$
\int_{0}^{t} I_{1}(\tau) \mathrm{d} \tau \leq C\|\tilde{g}\| \int_{0}^{t}\|\nabla w\|_{L^{2}}^{2} \mathrm{~d} \tau \leq C\|\tilde{g}\| D_{2}(t)^{2} .
$$

On the other hand, applying the Hölder inequality with $1 / n+1 / 2+1 / 2^{*}=1$ and using (2.3), we can estimate $I_{2}$ in (4.11) with $p=2$ as

$$
\begin{aligned}
I_{2} & =\int_{\mathbb{R}^{n}}\left|\bar{u}-u_{\infty}\right||\nabla w||\nabla \phi| \mathrm{d} x \\
& \leq C\left\|\bar{u}-u_{\infty}\right\|_{L^{n}}\|\nabla w\|_{L^{2}}\|\nabla \phi\|_{L^{2^{*}}} \leq C\|\tilde{g}\|\|\nabla w\|_{L^{2}}\|w-z\|_{L^{2}},
\end{aligned}
$$

which is a simpler version of (4.16). Thus we obtain

$$
\int_{0}^{t} I_{2}(\tau) \mathrm{d} \tau \leq C\|\tilde{g}\| \int_{0}^{t}\|\nabla w\|_{L^{2}}\|w-z\|_{L^{2}} \mathrm{~d} \tau \leq C\|\tilde{g}\| D_{2}(t)^{2}
$$

To estimate the term $J$ in (4.10) with $p=2$, we choose $r$ and $\lambda$ just in the same way as in (4.17) for $q_{1} \leq p \leq q$. Then the term $J$ is estimated as in (4.18) and we obtain

$$
J \leq C\|w\|_{L^{2}}^{\lambda}\|\nabla w\|_{L^{2}}^{2-\lambda}\|w-z\|_{L^{2}}^{\lambda}\|w-z\|_{L^{p}}^{1-\lambda} .
$$

Consequently, we have

$$
\int_{0}^{t} J(\tau) \mathrm{d} \tau \leq C E_{2}(t)^{\lambda} E_{p}(t)^{1-\lambda} \int_{0}^{t}\|\nabla w\|_{L^{2}}^{2-\lambda}\|w-z\|_{L^{2}}^{\lambda} \mathrm{d} \tau \leq C E_{2}(t)^{\lambda} E_{p}(t)^{1-\lambda} D_{2}(t)^{2} \leq C\left(E_{2}+E_{p}+D_{2}\right)(t)^{3} .
$$

Now, substituting all these estimates in (4.20), we have

$$
\|w(t)\|_{L^{2}}^{2}+\int_{0}^{t}\|\nabla w(\tau)\|_{L^{2}}^{2} \mathrm{~d} \tau+\int_{0}^{t} \int_{\mathbb{R}^{n}} w(w-z) \mathrm{d} x \mathrm{~d} \tau \leq C E_{0}^{2}+C\|\tilde{g}\| D_{2}(t)^{2}+C\left(E_{2}+E_{p}+D_{2}\right)(t)^{3} .
$$

Similarly, for $z$, we have

$$
\|z(t)\|_{L^{2}}^{2}+\int_{0}^{t}\|\nabla z(\tau)\|_{L^{2}}^{2} \mathrm{~d} \tau-\int_{0}^{t} \int_{\mathbb{R}^{n}} z(w-z) \mathrm{d} x \mathrm{~d} \tau \leq C E_{0}^{2}+C\|\tilde{g}\| D_{2}(t)^{2}+C\left(E_{2}+E_{p}+D_{2}\right)(t)^{3} .
$$

Adding these two inequalities, we obtain the desired estimate (4.19).

Step 4. Finally, we combine (4.13) and (4.19) to obtain

$$
X(t) \leq C E_{0}+C\left(\|\tilde{g}\|^{1 / 2}+\|\tilde{g}\|^{1 / p}\right) X(t)+C X(t)^{3 / 2}+C X(t)^{1+1 / p},
$$

where we put $X=E_{2}+E_{p}+D_{2}+D_{p}$. This inequality can be solved as $X(t) \leq C E_{0}$, provided that $E_{0}+\|\tilde{g}\|$ is suitably small. Thus we conclude that $\left(E_{2}+E_{p}+D_{2}+D_{p}\right)(t) \leq C E_{0}$, which gives the desired estimate (4.7). This completes the proof of Proposition 4.2.

Next we discuss the asymptotic stability of the stationary solution satisfying (4.1). We show the following decay estimate in $L^{p}$ for $p<n$. 
Theorem 4.3 ( $L^{p}$ decay estimate for $\left.p<n\right)$. Let $n \geq 3$ and $q_{1} \leq q<n$, where $q_{1}=\max (2, n / 2)$. Let $(w, z, \phi)$ be the global solution to the problem (4.3), (4.4) which is constructed in Theorem 4.1 for the initial data $\left(w_{0}, z_{0}\right) \in L^{2} \cap L^{q}$. If $E_{0}+\|\tilde{g}\|$ in Theorem 4.1 is suitably small, then we have

$$
\|(w, z)(t)\|_{L^{p}} \leq C E_{0}(1+t)^{-\gamma}
$$

for each $p$ with $2 \leq p \leq q$, where $\gamma=(n / 2)(1 / 2-1 / p)$. Moreover, we have

$$
\|\nabla \phi(t)\|_{L^{p^{\prime}}} \leq C E_{0}(1+t)^{-\gamma^{\prime}+1 / 2}
$$

for each $p^{\prime}$ with $2^{*} \leq p^{\prime} \leq q^{*}$, where $\gamma^{\prime}=(n / 2)\left(1 / 2-1 / p^{\prime}\right), 1 / 2^{*}=1 / 2-1 / n$ and $1 / q^{*}=1 / q-1 / n$.

Remark 4.4. The decay estimate (4.21) for $2 \leq p<n$ is just the same as the optimal $L^{p}-L^{2}$ decay estimate for the linear heat equation.

To prove Theorem 4.3, we introduce the following time weighted $L^{p}$ norms:

$$
\begin{aligned}
& E_{p, \beta}(t)=\sup _{0 \leq \tau \leq t}(1+\tau)^{\beta}\|(w, z)(\tau)\|_{L^{p}}, \\
& D_{p, \beta}(t)^{p}=\int_{0}^{t}(1+\tau)^{\beta p}\left(\left\|\nabla\left(|(w, z)|^{p / 2}\right)(\tau)\right\|_{L^{2}}^{2}+\|(w-z)(\tau)\|_{L^{p}}^{p}\right) \mathrm{d} \tau,
\end{aligned}
$$

where $2 \leq p<\infty$ and $\beta \geq 0$.

Proof of Theorem 4.3. Let $n \geq 3$ and $q_{1} \leq q<n$. By applying the time weighted $L^{p}$ energy method employed in $[8,10]$, we show that

$$
\left(E_{p, \beta}+D_{p, \beta}\right)(t)^{p} \leq C E_{0}^{p}(1+t)^{(\beta-\gamma) p}+C\left(E_{0}+\|\tilde{g}\|\right)\left(D_{p, \beta}+E_{p, \beta}\right)(t)^{p}
$$

for each $p$ with $q_{1} \leq p \leq q$ and for $\beta>\gamma$, where $\gamma=(n / 2)(1 / 2-1 / p)$. To prove (4.23), we multiply (4.9) by $(1+t)^{\beta p}$ and integrate the resulting inequality with respect to $t$. This yields

$$
\begin{aligned}
& (1+t)^{\beta p}\|w(t)\|_{L^{p}}^{p}+\int_{0}^{t}(1+\tau)^{\beta p}\left\|\nabla\left(|w|^{p / 2}\right)(\tau)\right\|_{L^{2}}^{2} \mathrm{~d} \tau+\int_{0}^{t}(1+\tau)^{\beta p} \int_{\mathbb{R}^{n}}|w|^{p-2} w(w-z) \mathrm{d} x \mathrm{~d} \tau \\
& \leq C\left\|w_{0}\right\|_{L^{p}}^{p}+C \int_{0}^{t}(1+\tau)^{\beta p-1}\|w(\tau)\|_{L^{p}}^{p} \mathrm{~d} \tau+C \int_{0}^{t}(1+\tau)^{\beta p}(I+J)(\tau) \mathrm{d} \tau .
\end{aligned}
$$

First we estimate the second term on the right hand side of (4.24). Applying (2.7) with $q=2$ and the Young inequality with $\gamma p /(1+\gamma p)+1 /(1+\gamma p)=1$, we obtain

$$
\begin{aligned}
& C \int_{0}^{t}(1+\tau)^{\beta p-1}\|w(\tau)\|_{L^{p}}^{p} \mathrm{~d} \tau \leq C \int_{0}^{t}(1+\tau)^{\beta p-1}\left\|\nabla\left(|w|^{p / 2}\right)(\tau)\right\|_{L^{2}}^{2 \gamma p /(1+\gamma p)}\|w(\tau)\|_{L^{2}}^{p /(1+\gamma p)} \mathrm{d} \tau \\
& \leq \delta \int_{0}^{t}(1+\tau)^{\beta p}\left\|\nabla\left(|w|^{p / 2}\right)(\tau)\right\|_{L^{2}}^{2} \mathrm{~d} \tau+C_{\delta} \int_{0}^{t}(1+\tau)^{(\beta-\gamma) p-1}\|w(\tau)\|_{L^{2}}^{p} \mathrm{~d} \tau \\
& \leq \delta \int_{0}^{t}(1+\tau)^{\beta p}\left\|\nabla\left(|w|^{p / 2}\right)(\tau)\right\|_{L^{2}}^{2} \mathrm{~d} \tau+C_{\delta} E_{0}^{p}(1+t)^{(\beta-\gamma) p}
\end{aligned}
$$

for any $\delta>0$ and $\beta>\gamma$, where $\gamma=(n / 2)(1 / 2-1 / p)$, and $C_{\delta}$ is a positive constant depending on $\delta$. Here we have used $\|w(t)\|_{L^{2}} \leq C E_{0}$ which is due to (4.6) with $p=2$.

On the other hand, for the term $I_{1}$, we have (4.14). Therefore the corresponding integral can be simply estimated as

$$
\int_{0}^{t}(1+\tau)^{\beta p} I_{1}(\tau) \mathrm{d} \tau \leq C\|\tilde{g}\| \int_{0}^{t}(1+\tau)^{\beta p}\left\|\nabla\left(|w|^{p / 2}\right)\right\|_{L^{2}}^{2} \mathrm{~d} \tau \leq C\|\tilde{g}\| D_{p, \beta}(t)^{p}
$$


To estimate the integral for $I_{2}$, we determine $s$ by

$$
\frac{1}{s}+\frac{1-2 / p}{2^{*}}+\frac{1}{2}+\frac{1}{p^{*}}=1
$$

where $1 / 2^{*}=1 / 2-1 / n$ and $1 / p^{*}=1 / p-1 / n$; here we used the restriction $p<n$. Notice that $s=n p / 2(p-1)$ and $s>2$. Then, applying the Hölder inequality with the relation (4.27) and using (2.6) and (2.3), similarly as in (4.16), we obtain

$$
\begin{aligned}
I_{2} & =(2 / p) \int_{\mathbb{R}^{n}}\left|\bar{u}-u_{\infty}\right||w|^{(p / 2)(1-2 / p)}\left|\nabla\left(|w|^{p / 2}\right)\right||\nabla \phi| \mathrm{d} x \\
& \leq C\left\|\bar{u}-u_{\infty}\right\|_{L^{s}}\left\|\left(|w|^{p / 2}\right)\right\|_{L^{2^{*}}}^{1-2 / p}\left\|\nabla\left(|w|^{p / 2}\right)\right\|_{L^{2}}\|\nabla \phi\|_{L^{p^{*}}} \\
& \leq C\|\tilde{g}\|\left\|\nabla\left(|w|^{p / 2}\right)\right\|_{L^{2}}^{2(1-1 / p)}\|w-z\|_{L^{p}} .
\end{aligned}
$$

Consequently, we have

$$
\int_{0}^{t}(1+\tau)^{\beta p} I_{2}(\tau) \mathrm{d} \tau \leq C\|\tilde{g}\| \int_{0}^{t}(1+\tau)^{\beta p}\left\|\nabla\left(|w|^{p / 2}\right)\right\|_{L^{2}}^{2(1-1 / p)}\|w-z\|_{L^{p}} \mathrm{~d} \tau \leq C\|\tilde{g}\| D_{p, \beta}(t)^{p}
$$

Finally, we estimate the integral for $J$. By using (4.18), we obtain

$$
\begin{aligned}
& \int_{0}^{t}(1+\tau)^{\beta p} J(\tau) \mathrm{d} \tau \leq C \int_{0}^{t}(1+\tau)^{\beta p}\|w\|_{L^{p}}^{\lambda p / 2}\left\|\nabla\left(|w|^{p / 2}\right)\right\|_{L^{2}}^{2-\lambda}\|w-z\|_{L^{2}}^{\lambda}\|w-z\|_{L^{p}}^{1-\lambda} \mathrm{d} \tau \\
& \leq C E_{p}(t)^{1-\lambda} E_{p, \beta}(t)^{\lambda p / 2} \int_{0}^{t}(1+\tau)^{\beta p(1-\lambda / 2)}\left\|\nabla\left(|w|^{p / 2}\right)\right\|_{L^{2}}^{2(1-\lambda / 2)}\|w-z\|_{L^{2}}^{\lambda} \mathrm{d} \tau \\
& \leq C E_{p}(t)^{1-\lambda} E_{p, \beta}(t)^{\lambda p / 2} D_{p, \beta}(t)^{(1-\lambda / 2) p} D_{2}(t)^{\lambda} \\
& \leq C\left(E_{p}+D_{2}\right)(t)\left(E_{p, \beta}+D_{p, \beta}\right)(t)^{p} \leq C E_{0}\left(E_{p, \beta}+D_{p, \beta}\right)(t)^{p},
\end{aligned}
$$

where we have used the Hölder inequality with $(1-\lambda / 2)+\lambda / 2=1$ and the estimate (4.6).

Substituting all these estimates in (4.24) and taking $\delta>0$ in (4.25) suitably small, we arrive at the inequality

$$
\begin{aligned}
& (1+t)^{\beta p}\|w(t)\|_{L^{p}}^{p}+\int_{0}^{t}(1+\tau)^{\beta p}\left\|\nabla\left(|w|^{p / 2}\right)(\tau)\right\|_{L^{2}}^{2} \mathrm{~d} \tau+\int_{0}^{t}(1+\tau)^{\beta p} \int_{\mathbb{R}^{n}}|w|^{p-2} w(w-z) \mathrm{d} x \mathrm{~d} \tau \\
& \leq C E_{0}^{p}(1+t)^{(\beta-\gamma) p}+C\left(E_{0}+\|\tilde{g}\|\right)\left(E_{p, \beta}+D_{p, \beta}\right)(t)^{p}
\end{aligned}
$$

for $\beta>\gamma$, where $\gamma=(n / 2)(1 / 2-1 / p)$. Similarly, for $z$, we have

$$
\begin{aligned}
& (1+t)^{\beta p}\|z(t)\|_{L^{p}}^{p}+\int_{0}^{t}(1+\tau)^{\beta p}\left\|\nabla\left(|z|^{p / 2}\right)(\tau)\right\|_{L^{2}}^{2} \mathrm{~d} \tau-\int_{0}^{t}(1+\tau)^{\beta p} \int_{\mathbb{R}^{n}}|z|^{p-2} z(w-z) \mathrm{d} x \mathrm{~d} \tau \\
& \leq C E_{0}^{p}(1+t)^{(\beta-\gamma) p}+C\left(E_{0}+\|\tilde{g}\|\right)\left(E_{p, \beta}+D_{p, \beta}\right)(t)^{p} .
\end{aligned}
$$

Adding these two inequalities, we reach the desired estimate (4.23).

Now we assume that $E_{0}+\|\tilde{g}\|$ is suitably small. Then (4.23) gives

$$
\left(E_{p, \beta}+D_{p, \beta}\right)(t) \leq C E_{0}(1+t)^{\beta-\gamma}
$$

for $q_{1} \leq p \leq q$ and $\beta>\gamma$, where $\gamma=(n / 2)(1 / 2-1 / p)$. In particular, we have

$$
\|(w, z)(t)\|_{L^{p}} \leq C E_{0}(1+t)^{-\gamma_{p}}
$$


for $q_{1} \leq p \leq q$, where we put $\gamma_{p}=(n / 2)(1 / 2-1 / p)$. Also, we have $\|(w, z)(t)\|_{L^{2}} \leq C E_{0}$ by (4.6), which shows that (4.31) holds true also for $p=2$. Therefore, applying the interpolation in $L^{p}$ with $1 / p=(1-\theta) / 2+\theta / q$ $(0 \leq \theta \leq 1)$ and using (4.31) with $p=2$ and $p=q$, we find that (4.31) holds true for each $p$ with $2 \leq p \leq q$. This proves the decay estimate (4.21).

Finally, we show (4.22). Let $2 \leq p \leq q$ and determine $p^{\prime}$ by $1 / p^{\prime}=1 / p-1 / n$. Notice that the range of $p^{\prime}$ is $2^{*} \leq p^{\prime} \leq q^{*}$. Using $(2.3)$ and (4.21), we obtain

$$
\|\nabla \phi(t)\|_{L^{p^{\prime}}} \leq C\|(w-z)(t)\|_{L^{p}} \leq C E_{0}(1+t)^{-\gamma}=C E_{0}(1+t)^{-\gamma^{\prime}+1 / 2},
$$

where $\gamma=(n / 2)(1 / 2-1 / p)$ and $\gamma^{\prime}=(n / 2)\left(1 / 2-1 / p^{\prime}\right)$; here we have used the relation $\gamma=\gamma^{\prime}-1 / 2$. Thus we have proved (4.22). This completes the proof of Theorem 4.3 .

When $p \geq n$, we have the following $L^{p}$ decay estimate in a weaker sense.

Theorem 4.5 ( $L^{p}$ decay estimate for $\left.p \geq n\right)$. Let $n \geq 3$ and $n \leq q<\infty$. Let $(w, z, \phi)$ be the global solution to the problem (4.3), (4.4) which is constructed in Theorem 4.1 for the initial data $\left(w_{0}, z_{0}\right) \in L^{2} \cap L^{q}$. If $E_{0}+\|\tilde{g}\|$ in Theorem 4.1 is suitably small, then we have the decay estimate (4.21) for each $p$ with $2 \leq p<n$. Moreover, for any small $\varepsilon>0$, we have

$$
\|(w, z)(t)\|_{L^{p}} \leq C_{\varepsilon} E_{0}(1+t)^{-\gamma_{n}+\varepsilon}
$$

for each $p$ with $n \leq p \leq q$, where $\gamma_{n}=(n / 2)(1 / 2-1 / n)$, and $C_{\varepsilon}$ is a positive constant depending on $\varepsilon$. Concerning $\nabla \phi$, we have the decay estimate (4.22) for each $p^{\prime}$ with $2^{*} \leq p^{\prime}<\infty$, where $1 / 2^{*}=1 / 2-1 / n$.

As a simple corollary of Theorem 4.5, we have:

Corollary 4.6 $\left(L^{\infty}\right.$ decay estimate for $\left.\nabla \phi\right)$. Assume the same conditions as in Theorem 4.5 for $n<q<\infty$. Then, for any small $\varepsilon>0$, we have

$$
\|\nabla \phi(t)\|_{L^{\infty}} \leq C_{\varepsilon} E_{0}(1+t)^{-\gamma_{n}+\varepsilon},
$$

where $\gamma_{n}=(n / 2)(1 / 2-1 / n)$, and $C_{\varepsilon}$ is a constant depending on $\varepsilon$.

The $L^{\infty}$ decay estimate (4.33) is verified as follows. We have $\|\nabla \phi(t)\|_{L^{p^{\prime}}} \leq C E_{0}(1+t)^{-\gamma^{\prime}+1 / 2}$ for $2^{*} \leq p^{\prime}<\infty$, where $\gamma^{\prime}=(n / 2)\left(1 / 2-1 / p^{\prime}\right)$. We choose $p^{\prime}=n / 2 \varepsilon$ such that $p^{\prime}>n$. For this choice of $p^{\prime}$, we see that $\gamma^{\prime}=n / 4-\varepsilon$ and hence $-\gamma^{\prime}+1 / 2=-\gamma_{n}+\varepsilon$, where $\gamma_{n}=(n / 2)(1 / 2-1 / n)$. Thus we have

$$
\|\nabla \phi(t)\|_{L^{p^{\prime}}} \leq C_{\varepsilon} E_{0}(1+t)^{-\gamma_{n}+\varepsilon},
$$

where $p^{\prime}=n / 2 \varepsilon>n$. Also it follows from (4.3c) and (4.32) that $\|\Delta \phi(t)\|_{L^{p}} \leq C_{\varepsilon} E_{0}(1+t)^{-\gamma_{n}+\varepsilon}$, which together with (2.4) gives

$$
\left\|\partial_{x}^{2} \phi(t)\right\|_{L^{p}} \leq C_{\varepsilon} E_{0}(1+t)^{-\gamma_{n}+\varepsilon}
$$

where $n<p \leq q$. On the other hand, as a simple version of the Gagliardo-Nirenberg inequality (2.5), we have

$$
\|u\|_{L^{\infty}} \leq C_{\varepsilon}\left\|\partial_{x} u\right\|_{L^{p}}^{\theta}\|u\|_{L^{p^{\prime}}}^{1-\theta},
$$

where $0=\theta(1 / p-1 / n)+(1-\theta) / p^{\prime}$. Substituting (4.34) and (4.35) into (4.36) for $u=\nabla \phi$, we get the desired decay estimate (4.33).

Proof of Theorem 4.5. Let $n \leq p \leq q$. It suffices to show the decay estimate (4.32). We use the time weighted $L^{p}$ energy inequality (4.24) which is valid even for $n \leq p \leq q$. The second term on the right hand side of (4.24) was already estimated in (4.25). In fact, we have

$$
C \int_{0}^{t}(1+\tau)^{\beta p-1}\|w(\tau)\|_{L^{p}}^{p} \mathrm{~d} \tau \leq \delta \int_{0}^{t}(1+\tau)^{\beta p}\left\|\nabla\left(|w|^{p / 2}\right)(\tau)\right\|_{L^{2}}^{2} \mathrm{~d} \tau+C_{\delta} E_{0}^{p}(1+t)^{\left(\beta-\gamma_{n}\right) p}
$$


for any $\delta>0$ and $\beta>\gamma_{n}$, where $\gamma_{n}=(n / 2)(1 / 2-1 / n)$, and $C_{\delta}$ is a positive constant depending on $\delta$. Also, the estimates (4.26) for $I_{1}$ and (4.29) for $J$ are valid for $n \leq p \leq q$.

To estimate the term $I_{2}$, we put $\rho=n /(1+2 \varepsilon)$, where $\varepsilon>0$ is so small that $2 \leq \rho<n$. For this choice of $\rho$, we determine $s$ by

$$
\frac{1}{s}+\frac{1-2 / p}{2^{*}}+\frac{1}{2}+\frac{1}{\rho^{*}}=1
$$

where $1 / 2^{*}=1 / 2-1 / n$ and $1 / \rho^{*}=1 / \rho-1 / n$. We see that $1 / s=(2 / n)(1-2 \varepsilon) / 2+(1-2 / n) / p$ and hence $2<s<p$. Also we find that $\gamma_{\rho}=\gamma_{n}-\varepsilon$, where $\gamma_{\rho}=(n / 2)(1 / 2-1 / \rho)$ and $\gamma_{n}=(n / 2)(1 / 2-1 / n)$. Now, similarly as in (4.28), we apply the Hölder inequality with the relation (4.38). Then, using (2.6) and (2.3), we obtain

$$
\begin{aligned}
I_{2} & \leq C\left\|\bar{u}-u_{\infty}\right\|_{L^{s}}\left\|\left(|w|^{p / 2}\right)\right\|_{L^{2^{*}}}^{1-2 / p}\left\|\nabla\left(|w|^{p / 2}\right)\right\|_{L^{2}}\|\nabla \phi\|_{L^{\rho^{*}}} \\
& \leq C_{\varepsilon}\|\tilde{g}\|\left\|\nabla\left(|w|^{p / 2}\right)\right\|_{L^{2}}^{2(1-1 / p)}\|w-z\|_{L^{\rho}},
\end{aligned}
$$

where $C_{\varepsilon}$ is a positive constant depending on $\varepsilon$. Consequently, we have

$$
\begin{aligned}
\int_{0}^{t}(1+\tau)^{\beta p} I_{2}(\tau) \mathrm{d} \tau & \leq C_{\varepsilon}\|\tilde{g}\| E_{\rho, \beta}(t)^{1-\rho / p} \int_{0}^{t}(1+\tau)^{\beta p(1-1 / p)+\beta \rho / p}\left\|\nabla\left(|w|^{p / 2}\right)\right\|_{L^{2}}^{2(1-1 / p)}\|w-z\|_{L^{\rho}}^{\rho / p} \mathrm{~d} \tau \\
& \leq C_{\varepsilon}\|\tilde{g}\| E_{\rho, \beta}(t)^{1-\rho / p} D_{p, \beta}(t)^{p-1} D_{\rho, \beta}(t)^{\rho / p} \\
& \leq C\|\tilde{g}\| D_{p, \beta}(t)^{p}+C_{\varepsilon}\|\tilde{g}\|\left(E_{\rho, \beta}+D_{\rho, \beta}\right)(t)^{p} \\
& \leq C\|\tilde{g}\| D_{p, \beta}(t)^{p}+C_{\varepsilon}\|\tilde{g}\| E_{0}^{p}(1+t)^{\left(\beta-\gamma_{n}+\varepsilon\right) p}
\end{aligned}
$$

for $\beta>\gamma_{\rho}$, where $\gamma_{\rho}=(n / 2)(1 / 2-1 / \rho)$ and $\gamma_{n}=(n / 2)(1 / 2-1 / n)$. Here we have used the Hölder inequality with $(1-1 / p)+1 / p=1$ and the estimate (4.30) with $p=\rho$, i.e., $\left(E_{\rho, \beta}+D_{\rho, \beta}\right)(t) \leq C_{\varepsilon}(1+t)^{\beta-\gamma_{\rho}}$ with the relation $\gamma_{\rho}=\gamma_{n}-\varepsilon$.

Substituting these estimates into (4.24) and taking $\delta>0$ in (4.37) suitably small, we obtain

$$
\begin{aligned}
& (1+t)^{\beta p}\|w(t)\|_{L^{p}}^{p}+\int_{0}^{t}(1+\tau)^{\beta p}\left\|\nabla\left(|w|^{p / 2}\right)(\tau)\right\|_{L^{2}}^{2} \mathrm{~d} \tau+\int_{0}^{t}(1+\tau)^{\beta p} \int_{\mathbb{R}^{n}}|w|^{p-2} w(w-z) \mathrm{d} x \mathrm{~d} \tau \\
& \leq C_{\varepsilon}(1+\|\tilde{g}\|) E_{0}^{p}(1+t)^{\left(\beta-\gamma_{n}+\varepsilon\right) p}+C\left(E_{0}+\|\tilde{g}\|\right)\left(E_{p, \beta}+D_{p, \beta}\right)(t)^{p}
\end{aligned}
$$

for $n \leq p \leq q$ and $\beta>\gamma_{n}$, where $\gamma_{n}=(n / 2)(1 / 2-1 / n)$. Similarly, for $z$, we have

$$
\begin{aligned}
& (1+t)^{\beta p}\|z(t)\|_{L^{p}}^{p}+\int_{0}^{t}(1+\tau)^{\beta p}\left\|\nabla\left(|z|^{p / 2}\right)(\tau)\right\|_{L^{2}}^{2} \mathrm{~d} \tau-\int_{0}^{t}(1+\tau)^{\beta p} \int_{\mathbb{R}^{n}}|z|^{p-2} z(w-z) \mathrm{d} x \mathrm{~d} \tau \\
& \leq C_{\varepsilon}(1+\|\tilde{g}\|) E_{0}^{p}(1+t)^{\left(\beta-\gamma_{n}+\varepsilon\right) p}+C\left(E_{0}+\|\tilde{g}\|\right)\left(E_{p, \beta}+D_{p, \beta}\right)(t)^{p} .
\end{aligned}
$$

Adding these two inequalities, we arrive at

$$
\left(E_{p, \beta}+D_{p, \beta}\right)(t)^{p} \leq C_{\varepsilon}(1+\|\tilde{g}\|) E_{0}^{p}(1+t)^{\left(\beta-\gamma_{n}+\varepsilon\right) p}+C\left(E_{0}+\|\tilde{g}\|\right)\left(E_{p, \beta}+D_{p, \beta}\right)(t)^{p}
$$

for $n \leq p \leq q$ and $\beta>\gamma_{n}$. This gives

$$
\left(E_{p, \beta}+D_{p, \beta}\right)(t) \leq C_{\varepsilon} E_{0}(1+t)^{\beta-\gamma_{n}+\varepsilon}
$$

for $n \leq p \leq q$ and $\beta>\gamma_{n}$, provided that $E_{0}+\|\tilde{g}\|$ is suitably small, where $\gamma_{n}=(n / 2)(1 / 2-1 / n)$. Thus we have the desired decay estimate (4.32). This completes the proof of Theorem 4.5. 
Finally in this section, we make a modification of the time weighted energy estimates (4.30) and (4.40), which will be used in the next section. We introduce

$$
\begin{aligned}
& \tilde{E}_{p, \beta}(t)=\sup _{0 \leq \tau \leq t} \tau^{\beta}\|(w, z)(\tau)\|_{L^{p}} \\
& \tilde{D}_{p, \beta}(t)^{p}=\int_{0}^{t} \tau^{\beta p}\left(\left\|\nabla\left(|(w, z)|^{p / 2}\right)(\tau)\right\|_{L^{2}}^{2}+\|(w-z)(\tau)\|_{L^{p}}^{p}\right) \mathrm{d} \tau .
\end{aligned}
$$

When $q_{1} \leq q<n$, we have

$$
\left(\tilde{E}_{p, \beta}+\tilde{D}_{p, \beta}\right)(t) \leq C E_{0} t^{\beta}(1+t)^{-\gamma}
$$

for $p=2$ and $q_{1} \leq p \leq q$ and for $\beta>\gamma$, where $\gamma=(n / 2)(1 / 2-1 / p)$; this is a modification of (4.30). On the other hand, if $n \leq q<\infty$, we have (4.41) for $p=2$ and $q_{1} \leq p<n$ and for $\beta>\gamma$. Also, for any $\varepsilon>0$, we have

$$
\left(\tilde{E}_{p, \beta}+\tilde{D}_{p, \beta}\right)(t) \leq C_{\epsilon} E_{0} t^{\beta}(1+t)^{-\gamma_{n}+\varepsilon}
$$

for $n \leq p \leq q$ and $\beta>\gamma_{n}$, where $\gamma_{n}=(n / 2)(1 / 2-1 / n)$; this is a modification of (4.40). These estimates are verified as follows. By using the uniform energy estimate (4.6), we have $\tau^{\beta}\|(w, z)(\tau)\|_{L^{p}} \leq C E_{0} t^{\beta}$ for $0 \leq \tau \leq t$. Also, using the time weighted energy estimate (4.30), we have $\tau^{\beta}\|(w, z)(\tau)\|_{L^{p}} \leq E_{p, \beta}(t) \leq C E_{0}(1+t)^{\beta-\gamma}$ for $0 \leq \tau \leq t$. Combining these two estimates, we obtain $\tilde{E}_{p, \beta}(t) \leq C E_{0} t^{\beta}(1+t)^{-\gamma}$. The other estimates in (4.41) and (4.42) can be shown in the same way.

\section{Decay estimates For DeRIVAtives}

In this section we show the decay estimates for derivatives of solutions by using the time weighted $L^{p}$ energy method again. When $p<n$, we have the following $L^{p}$ decay estimate for the derivatives.

Theorem 5.1 ( $L^{p}$ decay estimates for $p<n$ ). Let $n \geq 3$ and $q_{2} \leq q<n$, where $q_{2}=1+n / 2$. Let $(w, z, \phi)$ be the global solution to the problem (4.3), (4.4) which is constructed in Theorem 4.1 for the initial data $\left(w_{0}, z_{0}\right) \in L^{2} \cap L^{q}$. If $E_{0}+\|\tilde{g}\|$ in Theorem 4.1 is suitably small, then we have

$$
\left\|\partial_{x}(w, z)(t)\right\|_{L^{p}} \leq C E_{0}\left(1+t^{-1 / 2}\right)(1+t)^{-\gamma}
$$

for each $p$ with $2 \leq p \leq q$, where $\gamma=(n / 2)(1 / 2-1 / p)$. Moreover, we have

$$
\left\|\partial_{x}^{2} \phi(t)\right\|_{L^{p^{\prime}}} \leq C E_{0}\left(1+t^{-1 / 2}\right)(1+t)^{-\gamma^{\prime}+1 / 2}
$$

for each $p^{\prime}$ with $2^{*} \leq p^{\prime} \leq q^{*}$, where $\gamma^{\prime}=(n / 2)\left(1 / 2-1 / p^{\prime}\right), 1 / 2^{*}=1 / 2-1 / n$ and $1 / q^{*}=1 / q-1 / n$.

In order to prove Theorem 5.1, we introduce the following time weighted norms:

$$
\begin{aligned}
& \dot{E}_{p, \beta}(t)=\sup _{0 \leq \tau \leq t} \tau^{\beta}\left\|\partial_{x}(w, z)(\tau)\right\|_{L^{p}}, \\
& \dot{D}_{p, \beta}(t)^{p}=\int_{0}^{t} \tau^{\beta p}\left(\left\|\nabla\left(\left|\partial_{x}(w, z)\right|^{p / 2}\right)(\tau)\right\|_{L^{2}}^{2}+\left\|\partial_{x}(w-z)(\tau)\right\|_{L^{p}}^{p}\right) \mathrm{d} \tau .
\end{aligned}
$$

Proof of Theorem 5.1. Let $n \geq 3$. As in the proof of Proposition 4.2, we divide our proof into four parts.

Step 1. Let $2 \leq p \leq q$. We derive the time weighted $L^{p}$ energy inequality for derivatives of solutions to the problem (4.3), (4.4). We differentiate (4.3a) with respect to $x_{i}$ to obtain

$$
w_{i, t}-\Delta w_{i}+u_{\infty} \Delta \phi_{i}=-\partial_{x_{i}}\left(\nabla \cdot f_{1}+\nabla \cdot(w \nabla \phi)\right),
$$


where we simply wrote as $w_{i}=\partial_{x_{i}} w, z_{i}=\partial_{x_{i}} z$ and $\phi_{i}=\partial_{x_{i}} \phi$ for $i=1, \ldots, n$. We multiply (5.3) by $\left|w_{i}\right|^{p-2} w_{i}$. This gives

$$
\begin{aligned}
\frac{1}{p}\left(\left|w_{i}\right|^{p}\right)_{t}+ & c_{0}\left|\nabla\left(\left|w_{i}\right|^{p / 2}\right)\right|^{2}+u_{\infty}\left|w_{i}\right|^{p-2} w_{i}\left(w_{i}-z_{i}\right)-\nabla \cdot\left(\left|w_{i}\right|^{p-2} w_{i} \nabla w_{i}\right) \\
=- & \partial_{x_{i}}\left(\left|w_{i}\right|^{p-2} w_{i} \nabla \cdot f_{1}+\left|w_{i}\right|^{p-2} w_{i} \nabla \cdot(w \nabla \phi)\right) \\
& +(p-1)\left(\left|w_{i}\right|^{p-2} \partial_{x_{i}} w_{i} \nabla \cdot f_{1}+\left|w_{i}\right|^{p-2} \partial_{x_{i}} w_{i} \nabla \cdot(w \nabla \phi)\right),
\end{aligned}
$$

where $c_{0}=4(p-1) / p^{2}$. Here we used (4.3c). We integrate (5.4) over $\mathbb{R}^{n}$, obtaining

$$
\frac{1}{p} \frac{\mathrm{d}}{\mathrm{d} t}\left\|w_{i}\right\|_{L^{p}}^{p}+c_{0}\left\|\nabla\left(\left|w_{i}\right|^{p / 2}\right)\right\|_{L^{2}}^{2}+u_{\infty} \int_{\mathbb{R}^{n}}\left|w_{i}\right|^{p-2} w_{i}\left(w_{i}-z_{i}\right) \mathrm{d} x \leq(p-1)\left(I^{\prime}+J^{\prime}\right),
$$

where

$$
I^{\prime}=\int_{\mathbb{R}^{n}}\left|w_{i}\right|^{p-2}\left|\partial_{x_{i}} w_{i}\right|\left|\nabla \cdot f_{1}\right| \mathrm{d} x, \quad J^{\prime}=\int_{\mathbb{R}^{n}}\left|w_{i}\right|^{p-2}\left|\partial_{x_{i}} w_{i}\right||\nabla \cdot(w \nabla \phi)| \mathrm{d} x
$$

It follows from (4.5) that $I^{\prime} \leq \sum_{j=1}^{4} I_{j}^{\prime}$ and $J^{\prime} \leq J_{1}^{\prime}+J_{2}^{\prime}$, where

$$
\begin{array}{rlrl}
I_{1}^{\prime} & =\int_{\mathbb{R}^{n}}|\nabla \bar{\psi}||\nabla w|\left|w_{i}\right|^{p-2}\left|\partial_{x_{i}} w_{i}\right| \mathrm{d} x, & I_{2}^{\prime} & =\int_{\mathbb{R}^{n}}|\Delta \bar{\psi}||w|\left|w_{i}\right|^{p-2}\left|\partial_{x_{i}} w_{i}\right| \mathrm{d} x, \\
I_{3}^{\prime}=\int_{\mathbb{R}^{n}}|\nabla \bar{u}|\left|w_{i}\right|^{p-2}\left|\partial_{x_{i}} w_{i}\right||\nabla \phi| \mathrm{d} x, & I_{4}^{\prime}=\int_{\mathbb{R}^{n}}\left|\bar{u}-u_{\infty}\right|\left|w_{i}\right|^{p-2}\left|\partial_{x_{i}} w_{i}\right||w-z| \mathrm{d} x, \\
J_{1}^{\prime}=\int_{\mathbb{R}^{n}}|\nabla w|\left|w_{i}\right|^{p-2}\left|\partial_{x_{i}} w_{i}\right||\nabla \phi| \mathrm{d} x, & J_{2}^{\prime}=\int_{\mathbb{R}^{n}}|w|\left|w_{i}\right|^{p-2}\left|\partial_{x_{i}} w_{i}\right||w-z| \mathrm{d} x .
\end{array}
$$

Here we again used (4.3c). We multiply (5.5) by $t^{\beta p}$, where $\beta>0$ is a suitably large number which will be specified later, and integrate the resulting equation with respect to $t$. This yields

$$
\begin{aligned}
& t^{\beta p}\left\|w_{i}(t)\right\|_{L^{p}}^{p}+\int_{0}^{t} \tau^{\beta p}\left\|\nabla\left(\left|w_{i}\right|^{p / 2}\right)(\tau)\right\|_{L^{2}}^{2} \mathrm{~d} \tau+\int_{0}^{t} \tau^{\beta p} \int_{\mathbb{R}^{n}}\left|w_{i}\right|^{p-2} w_{i}\left(w_{i}-z_{i}\right) \mathrm{d} x \mathrm{~d} \tau \\
& \leq C \int_{0}^{t} \tau^{\beta p-1}\left\|w_{i}(\tau)\right\|_{L^{p}}^{p} \mathrm{~d} \tau+C \int_{0}^{t} \tau^{\beta p}\left(I^{\prime}+J^{\prime}\right)(\tau) \mathrm{d} \tau .
\end{aligned}
$$

We have a similar energy inequality also for $z_{i}$.

Step 2. Let $q_{2} \leq p \leq q$. We show the following time weighted $L^{p}$ energy estimate:

$$
\left(\dot{E}_{p, \beta}+\dot{D}_{p, \beta}\right)(t) \leq C E_{0} t^{\beta}\left(1+t^{-1 / 2}\right)(1+t)^{-\gamma}
$$

for $\beta>\gamma+1 / 2$, where $\gamma=(n / 2)(1 / 2-1 / p)$. This gives the desired decay estimate (5.1) for $q_{2} \leq p \leq q$. To prove (5.9), we need to estimate the right hand side of (5.8). For the first term, by applying the estimate $(2.8)$ and the Young inequality with $p /(p+2)+2 /(p+2)=1$, we obtain

$$
\begin{aligned}
& C \int_{0}^{t} \tau^{\beta p-1}\left\|w_{i}(\tau)\right\|_{L^{p}}^{p} \mathrm{~d} \tau \leq C \int_{0}^{t} \tau^{\beta p-1}\left\|\nabla\left(\left|w_{i}\right|^{p / 2}\right)(\tau)\right\|_{L^{2}}^{2 p /(p+2)}\|w(\tau)\|_{L^{p}}^{2 p /(p+2)} \mathrm{d} \tau \\
& \leq \delta \int_{0}^{t} \tau^{\beta p}\left\|\nabla\left(\left|w_{i}\right|^{p / 2}\right)(\tau)\right\|_{L^{2}}^{2} \mathrm{~d} \tau+C_{\delta} \int_{0}^{t} \tau^{(\beta-1 / 2) p-1}\|w(\tau)\|_{L^{p}}^{p} \mathrm{~d} \tau \\
& \leq \delta \int_{0}^{t} \tau^{\beta p}\left\|\nabla\left(\left|w_{i}\right|^{p / 2}\right)(\tau)\right\|_{L^{2}}^{2} \mathrm{~d} \tau+C_{\delta} E_{0}^{p} t^{(\beta-1 / 2) p}(1+t)^{-\gamma p}
\end{aligned}
$$


for any $\delta>0$ and $\beta>\gamma+1 / 2$, where $\gamma=(n / 2)(1 / 2-1 / p)$, and $C_{\delta}$ is a positive constant depending on $\delta$. Here we have used $\|w(t)\|_{L^{p}} \leq C E_{0}(1+t)^{-\gamma}$ in (4.21) and the inequality $\int_{0}^{t} \tau^{(\beta-1 / 2) p-1}(1+\tau)^{-\gamma p} \mathrm{~d} \tau \leq$ $C t^{(\beta-1 / 2) p}(1+t)^{-\gamma p}$ for $\beta>\gamma+1 / 2$.

Next we estimate the integral for $I^{\prime}$ which is divided into four terms in (5.7). We see that the term $I_{1}^{\prime}$ is similar to $I_{1}$ in (4.11). Therefore, applying the Hölder inequality with $1 / n+1 / 2^{*}+1 / 2=1$ and using (2.6), we have

$$
\begin{aligned}
I_{1}^{\prime} & \leq C \int_{\mathbb{R}^{n}}|\nabla \bar{\psi}|\left|\partial_{x} w\right|^{p / 2}\left|\nabla\left(\left|\partial_{x} w\right|^{p / 2}\right)\right| \mathrm{d} x \\
& \leq C\|\nabla \bar{\psi}\|_{L^{n}}\left\|\left(\left|\partial_{x} w\right|^{p / 2}\right)\right\|_{L^{2^{*}}}\left\|\nabla\left(\left|\partial_{x} w\right|^{p / 2}\right)\right\|_{L^{2}} \leq C\|\tilde{g}\|\left\|\nabla\left(\left|\partial_{x} w\right|^{p / 2}\right)\right\|_{L^{2}}^{2} .
\end{aligned}
$$

Thus we obtain

$$
\int_{0}^{t} \tau^{\beta p} I_{1}^{\prime}(\tau) \mathrm{d} \tau \leq C\|\tilde{g}\| \int_{0}^{t} \tau^{\beta p}\left\|\nabla\left(\left|\partial_{x} w\right|^{p / 2}\right)\right\|_{L^{2}}^{2} \mathrm{~d} \tau \leq C\|\tilde{g}\| \dot{D}_{p, \beta}(t)^{p} .
$$

Similarly, applying the Hölder inequality with $1 / n+(2 / p) / 2^{*}+(1-2 / p) / 2^{*}+1 / 2=1$ and using (2.6), we can estimate the term $I_{2}^{\prime}$ as

$$
\begin{aligned}
I_{2}^{\prime} & \leq C \int_{\mathbb{R}^{n}}|\Delta \bar{\psi}||w|^{(p / 2)(2 / p)}\left|\partial_{x} w\right|^{(p / 2)(1-2 / p)}\left|\nabla\left(\left|\partial_{x} w\right|^{p / 2}\right)\right| \mathrm{d} x \\
& \leq C\|\Delta \bar{\psi}\|_{L^{n}}\left\|\left(|w|^{p / 2}\right)\right\|_{L^{2^{*}}}^{2 / p}\left\|\left(\left|\partial_{x} w\right|^{p / 2}\right)\right\|_{L^{2^{*}}}^{1-2 / p}\left\|\nabla\left(\left|\partial_{x} w\right|^{p / 2}\right)\right\|_{L^{2}} \\
& \leq C\|\tilde{g}\|\left\|\nabla\left(|w|^{p / 2}\right)\right\|_{L^{2}}^{2 / p}\left\|\nabla\left(\left|\partial_{x} w\right|^{p / 2}\right)\right\|_{L^{2}}^{2(1-1 / p)}
\end{aligned}
$$

Consequently, we obtain

$$
\begin{aligned}
\int_{0}^{t} \tau^{\beta p} I_{2}^{\prime}(\tau) \mathrm{d} \tau & \leq C\|\tilde{g}\| \int_{0}^{t} \tau^{\beta p}\left\|\nabla\left(|w|^{p / 2}\right)\right\|_{L^{2}}^{2 / p}\left\|\nabla\left(\left|\partial_{x} w\right|^{p / 2}\right)\right\|_{L^{2}}^{2(1-1 / p)} \mathrm{d} \tau \\
& \leq C\|\tilde{g}\| \tilde{D}_{p, \beta}(t) \dot{D}_{p, \beta}(t)^{p-1} \leq C\|\tilde{g}\| \dot{D}_{p, \beta}(t)^{p}+C\|\tilde{g}\| E_{0}^{p} t^{\beta p}(1+t)^{-\gamma p}
\end{aligned}
$$

for $\beta>\gamma$, where $\gamma=(n / 2)(1 / 2-1 / p)$. Here we have used the Hölder inequality with $1 / p+(p-1) / p=1$ and the estimate $\tilde{D}_{p, \beta}(t) \leq C E_{0} t^{\beta}(1+t)^{-\gamma}$ in $(4.41)$.

On the other hand, to estimate the term $I_{3}^{\prime}$ which is similar to $I_{2}$ in (4.11), we determine $s$ with $s>2$ by the relation $1 / s+(1-2 / p) / 2^{*}+1 / 2+1 / p^{*}=1$ in (4.27). Then, applying the Hölder inequality with this relation and using (2.6) and (2.3), we obtain

$$
\begin{aligned}
I_{3}^{\prime} & \leq C \int_{\mathbb{R}^{n}}|\nabla \bar{u}|\left|\partial_{x} w\right|^{(p / 2)(1-2 / p)}\left|\nabla\left(\left|\partial_{x} w\right|^{p / 2}\right)\right||\nabla \phi| \mathrm{d} x \\
& \leq C\|\nabla \bar{u}\|_{L^{s}}\left\|\left(\left|\partial_{x} w\right|^{p / 2}\right)\right\|_{L^{2^{*}}}^{1-2 / p}\left\|\nabla\left(\left|\partial_{x} w\right|^{p / 2}\right)\right\|_{L^{2}}\|\nabla \phi\|_{L^{p^{*}}} \\
& \leq C\|\tilde{g}\|\left\|\nabla\left(\left|\partial_{x} w\right|^{p / 2}\right)\right\|_{L^{2}}^{2(1-1 / p)}\|w-z\|_{L^{p}} .
\end{aligned}
$$

Consequently, we have

$$
\begin{aligned}
\int_{0}^{t} \tau^{\beta p} I_{3}^{\prime}(\tau) \mathrm{d} \tau & \leq C\|\tilde{g}\| \int_{0}^{t} \tau^{\beta p}\left\|\nabla\left(\left|\partial_{x} w\right|^{p / 2}\right)\right\|_{L^{2}}^{2(1-1 / p)}\|w-z\|_{L^{p}} \mathrm{~d} \tau \\
& \leq C\|\tilde{g}\| \dot{D}_{p, \beta}(t)^{p-1} \tilde{D}_{p, \beta}(t) \leq C\|\tilde{g}\| \dot{D}_{p, \beta}(t)^{p}+C\|\tilde{g}\| E_{0}^{p} t^{\beta p}(1+t)^{-\gamma p}
\end{aligned}
$$


for $\beta>\gamma$, where we used the Hölder inequality with $(p-1) / p+1 / p=1$ and (4.41). Also, the term $I_{4}^{\prime}$ is treated similarly. In fact, applying the Hölder inequality with the relation $1 / s+(1-2 / p) / 2^{*}+1 / 2+1 / p^{*}=1$ in $(4.27)$ and using (2.6), we obtain

$$
\begin{aligned}
I_{4}^{\prime} & \leq C \int_{\mathbb{R}^{n}}\left|\bar{u}-u_{\infty}\right|\left|\partial_{x} w\right|^{(p / 2)(1-2 / p)}\left|\nabla\left(\left|\partial_{x} w\right|^{p / 2}\right)\right||w-z| \mathrm{d} x \\
& \leq C\left\|\bar{u}-u_{\infty}\right\|_{L^{s}}\left\|\left(\left|\partial_{x} w\right|^{p / 2}\right)\right\|_{L^{2^{*}}}^{1-2 / p}\left\|\nabla\left(\left|\partial_{x} w\right|^{p / 2}\right)\right\|_{L^{2}}\|w-z\|_{L^{p^{*}}} \\
& \leq C\|\tilde{g}\|\left\|\nabla\left(\left|\partial_{x} w\right|^{p / 2}\right)\right\|_{L^{2}}^{2(1-1 / p)}\left\|\partial_{x}(w-z)\right\|_{L^{p}} .
\end{aligned}
$$

Consequently, we have

$$
\int_{0}^{t} \tau^{\beta p} I_{4}^{\prime}(\tau) \mathrm{d} \tau \leq C\|\tilde{g}\| \int_{0}^{t} \tau^{\beta p}\left\|\nabla\left(\left|\partial_{x} w\right|^{p / 2}\right)\right\|_{L^{2}}^{2(1-1 / p)}\left\|\partial_{x}(w-z)\right\|_{L^{p}} \mathrm{~d} \tau \leq C\|\tilde{g}\| \dot{D}_{p, \beta}(t)^{p} .
$$

Finally, we estimate the integral for $J^{\prime}$ which is divided into two terms in (5.7). We see that the term $J_{1}^{\prime}$ is similar to $J$ in (4.10). Therefore, to estimate $J_{1}^{\prime}$, we determine $r$ and $\lambda$ by the relations in (4.17); here the restriction $p \geq n / 2$ is used. Then, applying the Hölder inequality with $\lambda / 2+(1-\lambda) / 2^{*}+1 / 2+1 / r^{*}=1$ in (4.17) and using (2.6) and (2.3), we have

$$
\begin{aligned}
J_{1}^{\prime} & \leq C \int_{\mathbb{R}^{n}}\left|\partial_{x} w\right|^{\lambda p / 2}\left|\partial_{x} w\right|^{(1-\lambda) p / 2}\left|\nabla\left(\left|\partial_{x} w\right|^{p / 2}\right)\right||\nabla \phi| \mathrm{d} x \\
& \leq C\left\|\left(\left|\partial_{x} w\right|^{p / 2}\right)\right\|_{L^{2}}^{\lambda}\left\|\left(\left|\partial_{x} w\right|^{p / 2}\right)\right\|_{L^{2^{*}}}^{1-\lambda}\left\|\nabla\left(\left|\partial_{x} w\right|^{p / 2}\right)\right\|_{L^{2}}\|\nabla \phi\|_{L^{r^{*}}} \\
& \leq C\left\|\partial_{x} w\right\|_{L^{p}}^{\lambda p / 2}\left\|\nabla\left(\left|\partial_{x} w\right|^{p / 2}\right)\right\|_{L^{2}}^{2-\lambda}\|w-z\|_{L^{2}}^{\lambda}\|w-z\|_{L^{p}}^{1-\lambda},
\end{aligned}
$$

where we have used the interpolation in $L^{r}$ with the relation $1 / r=\lambda / 2+(1-\lambda) / p$ in (4.17). Consequently, in the same way as in (4.29), we obtain

$$
\begin{aligned}
\int_{0}^{t} \tau^{\beta p} J_{1}^{\prime}(\tau) \mathrm{d} \tau & \leq C \int_{0}^{t} \tau^{\beta p}\left\|\partial_{x} w\right\|_{L^{p}}^{\lambda p / 2}\left\|\nabla\left(\left|\partial_{x} w\right|^{p / 2}\right)\right\|_{L^{2}}^{2-\lambda}\|w-z\|_{L^{2}}^{\lambda}\|w-z\|_{L^{p}}^{1-\lambda} \mathrm{d} \tau \\
& \leq C E_{p}(t)^{1-\lambda} \dot{E}_{p, \beta}(t)^{\lambda p / 2} \int_{0}^{t} \tau^{\beta p(1-\lambda / 2)}\left\|\nabla\left(\left|\partial_{x} w\right|^{p / 2}\right)\right\|_{L^{2}}^{2(1-\lambda / 2)}\|w-z\|_{L^{2}}^{\lambda} \mathrm{d} \tau \\
& \leq C E_{p}(t)^{1-\lambda} \dot{E}_{p, \beta}(t)^{\lambda p / 2} \dot{D}_{p, \beta}(t)^{(1-\lambda / 2) p} D_{2}(t)^{\lambda} \leq C E_{0}\left(\dot{E}_{p, \beta}+\dot{D}_{p, \beta}\right)(t)^{p},
\end{aligned}
$$

where we used the Hölder inequality with $(2-\lambda) / 2+\lambda / 2=1$ and (4.6).

On the other hand, the term $J_{2}^{\prime}$ is similar to $I_{4}^{\prime}$. So we determine $s$ by the relation $1 / s+(1-2 / p) / 2^{*}+1 / 2+$ $1 / p^{*}=1$ in (4.27). This $s$ satisfies $2<s \leq p$ for $q_{2} \leq p<n$; the restriction $p \geq q_{2}=n / 2+1$ is used here. Then, applying the Hölder inequality with the relation in (4.27) and using (2.6), we obtain

$$
\begin{aligned}
J_{2}^{\prime} & \leq C \int_{\mathbb{R}^{n}}|w|\left|\partial_{x} w\right|^{(p / 2)(1-2 / p)}\left|\nabla\left(\left|\partial_{x} w\right|^{p / 2}\right)\right||w-z| \mathrm{d} x \\
& \leq C\|w\|_{L^{s}}\left\|\left(\left|\partial_{x} w\right|^{p / 2}\right)\right\|_{L^{2^{*}}}^{1-2 / p}\left\|\nabla\left(\left|\partial_{x} w\right|^{p / 2}\right)\right\|_{L^{2}}\|w-z\|_{L^{p^{*}}} \\
& \leq C\|w\|_{L^{s}}\left\|\nabla\left(\left|\partial_{x} w\right|^{p / 2}\right)\right\|_{L^{2}}^{2(1-1 / p)}\left\|\partial_{x}(w-z)\right\|_{L^{p}} .
\end{aligned}
$$

Consequently, we have

$$
\begin{aligned}
\int_{0}^{t} \tau^{\beta p} J_{2}^{\prime}(\tau) \mathrm{d} \tau & \leq C E_{s}(t) \int_{0}^{t} \tau^{\beta p}\left\|\nabla\left(\left|\partial_{x} w\right|^{p / 2}\right)\right\|_{L^{2}}^{2(1-1 / p)}\left\|\partial_{x}(w-z)\right\|_{L^{p}} \mathrm{~d} \tau \\
& \leq C E_{s}(t) \dot{D}_{p, \beta}(t)^{p} \leq C E_{0} \dot{D}_{p, \beta}(t)^{p}
\end{aligned}
$$

where we used (4.6). 
Substituting all these estimates in (5.8) and taking $\delta>0$ in (5.10) suitably small, we arrive at the inequality

$$
\begin{aligned}
& t^{\beta p}\left\|\partial_{x} w(t)\right\|_{L^{p}}^{p}+\int_{0}^{t} \tau^{\beta p}\left\|\nabla\left(\left|\partial_{x} w\right|^{p / 2}\right)(\tau)\right\|_{L^{2}}^{2} \mathrm{~d} \tau+\int_{0}^{t} \tau^{\beta p} \int_{\mathbb{R}^{n}}\left|\partial_{x} w\right|^{p-2} \partial_{x}(w-z) \mathrm{d} x \mathrm{~d} \tau \\
& \leq C(1+\|\tilde{g}\|) E_{0}^{p} t^{\beta p}\left(1+t^{-p / 2}\right)(1+t)^{-\gamma p}+C\left(E_{0}+\|\tilde{g}\|\right)\left(\dot{E}_{p, \beta}+\dot{D}_{p, \beta}\right)(t)^{p}
\end{aligned}
$$

for $\beta>\gamma+1 / 2$, where $\gamma=(n / 2)(1 / 2-1 / p)$. We have the corresponding energy inequality also for $\partial_{x} z$. Adding these two inequalities, we obtain

$$
\left(\dot{E}_{p, \beta}+\dot{D}_{p, \beta}\right)(t)^{p} \leq C(1+\|\tilde{g}\|) E_{0}^{p} t^{\beta p}\left(1+t^{-p / 2}\right)(1+t)^{-\gamma p}+C\left(E_{0}+\|\tilde{g}\|\right)\left(\dot{E}_{p, \beta}+\dot{D}_{p, \beta}\right)(t)^{p},
$$

where $q_{2} \leq p \leq q, \beta>\gamma+1 / 2$ and $\gamma=(n / 2)(1 / 2-1 / n)$. This gives the desired estimate (5.9) for suitably small $E_{0}+\|\tilde{g}\|$.

Step 3. We show that the time weighted energy estimate (5.9) holds true also for $p=2$. Namely, we show that

$$
\left(\dot{E}_{2, \beta}+\dot{D}_{2, \beta}\right)(t) \leq C E_{0} t^{\beta}\left(1+t^{-1 / 2}\right)
$$

for $\beta>1 / 2$. This gives the estimate (5.1) for $p=2$. To prove (5.18), we need to estimate the right hand side of (5.8) for $p=2$, in which $I^{\prime}$ and $J^{\prime}$ are given in (5.6) with $p=2$. We see that the estimate (5.10) is useful for $p=2$. Also, all the estimates for $I_{1}^{\prime}, \ldots, I_{4}^{\prime}$ in Step 2 are valid even for $p=2$. To estimate the term $J_{1}^{\prime}$ for $p=2$, we again use $r$ and $\lambda$ determined by (4.17), where $p \geq n / 2$. Then we can modify the estimate (5.15) in Step 2 as

$$
J_{1}^{\prime} \leq C\left\|\partial_{x} w\right\|_{L^{2}}^{\lambda}\left\|\nabla \partial_{x} w\right\|_{L^{2}}^{2-\lambda}\|w-z\|_{L^{2}}^{\lambda}\|w-z\|_{L^{p}}^{1-\lambda} .
$$

Consequently, as a modification of (5.16), we have

$$
\begin{aligned}
& \int_{0}^{t} \tau^{2 \beta} J_{1}^{\prime}(\tau) \mathrm{d} \tau \leq C E_{p}(t)^{1-\lambda} \dot{E}_{2, \beta}(t)^{\lambda} \int_{0}^{t} \tau^{2 \beta(1-\lambda / 2)}\left\|\nabla \partial_{x} w\right\|_{L^{2}}^{2(1-\lambda / 2)}\|w-z\|_{L^{2}}^{\lambda} \mathrm{d} \tau \\
& \leq C E_{p}(t)^{1-\lambda} \dot{E}_{2, \beta}(t)^{\lambda} \dot{D}_{2, \beta}(t)^{2-\lambda} D_{2}(t)^{\lambda} \leq C E_{0}\left(\dot{E}_{2, \beta}+\dot{D}_{2, \beta}\right)(t)^{2},
\end{aligned}
$$

where we used the Hölder inequality with $(2-\lambda) / 2+\lambda / 2=1$ and (4.6).

Finally, we estimate the term $J_{2}^{\prime}$ for $p=2$. We also use $r$ and $\lambda$ in (4.17), and then define $s$ by

$$
\frac{1}{s}=\frac{\lambda}{2}+\frac{1-\lambda}{2^{*}}
$$

We find that this $s$ satisfies $2<s<n$ for $p_{2} \leq p<n$. Therefore the first relation $\lambda / 2+(1-\lambda) / 2^{*}+1 / 2+1 / r^{*}=1$ in (4.17) is rewritten as $1 / s^{*}+1 / 2+1 / r=1$, where $s^{*}=1 / s-1 / n$. Applying the Hölder inequality with this last relation and using $(2.6)$, we have

$$
\begin{aligned}
J_{2}^{\prime} & \leq C \int_{\mathbb{R}^{n}}|w|\left|\nabla \partial_{x} w\right||w-z| \mathrm{d} x \\
& \leq C\|w\|_{L^{s^{*}}}\left\|\nabla \partial_{x} w\right\|_{L^{2}}\|w-z\|_{L^{r}} \leq C\left\|\partial_{x} w\right\|_{L^{s}}\left\|\nabla \partial_{x} w\right\|_{L^{2}}\|w-z\|_{L^{r}} .
\end{aligned}
$$

Moreover, using the interpolation in $L^{s}$ with the relation (5.21), we have $\left\|\partial_{x} w\right\|_{L^{s}} \leq\left\|\partial_{x} w\right\|_{L^{2}}^{\lambda}\left\|\partial_{x} w\right\|_{L^{2^{*}}}^{1-\lambda} \leq$ $C\left\|\partial_{x} w\right\|_{L^{2}}^{\lambda}\left\|\nabla \partial_{x} w\right\|_{L^{2}}^{1-\lambda}$, where we used (2.6). Similarly, using the interpolation with $1 / r=\lambda / 2+(1-\lambda) / p$ in (4.17), we have $\|w-z\|_{L^{r}} \leq\|w-z\|_{L^{2}}^{\lambda}\|w-z\|_{L^{p}}^{1-\lambda}$. Consequently, we obtain

$$
J_{2}^{\prime} \leq C\left\|\partial_{x} w\right\|_{L^{2}}^{\lambda}\left\|\nabla \partial_{x} w\right\|_{L^{2}}^{1-\lambda}\left\|\nabla \partial_{x} w\right\|_{L^{2}}\|w-z\|_{L^{2}}^{\lambda}\|w-z\|_{L^{p}}^{1-\lambda},
$$

which is just the same as (5.19). Therefore, just in the same way as in (5.20), we get

$$
\int_{0}^{t} \tau^{2 \beta} J_{2}^{\prime}(\tau) \mathrm{d} \tau \leq C E_{0}\left(\dot{E}_{2, \beta}+\dot{D}_{2, \beta}\right)(t)^{2} .
$$


Substituting all these estimates in (5.8) for $p=2$, we arrive at the inequality

$$
\begin{aligned}
& \left.t^{2 \beta}\left\|\partial_{x} w(t)\right\|_{L^{p}}^{p}+\int_{0}^{t} \tau^{2 \beta} \| \nabla \partial_{x} w\right)(\tau) \|_{L^{2}}^{2} \mathrm{~d} \tau+\int_{0}^{t} \tau^{2 \beta} \int_{\mathbb{R}^{n}} \partial_{x}(w-z) \mathrm{d} x \mathrm{~d} \tau \\
& \leq C(1+\|\tilde{g}\|) E_{0}^{2} t^{2 \beta}\left(1+t^{-1}\right)+C\left(E_{0}+\|\tilde{g}\|\right)\left(\dot{E}_{2, \beta}+\dot{D}_{2, \beta}\right)(t)^{2}
\end{aligned}
$$

for $\beta>1 / 2$. Combining this estimate with the corresponding inequality for $\partial_{x} z$, we obtain

$$
\left(\dot{E}_{2, \beta}+\dot{D}_{2, \beta}\right)(t)^{2} \leq C(1+\|\tilde{g}\|) E_{0}^{2} t^{2 \beta}\left(1+t^{-1}\right)+C\left(E_{0}+\|\tilde{g}\|\right)\left(\dot{E}_{2, \beta}+\dot{D}_{2, \beta}\right)(t)^{2}
$$

for $\beta>1 / 2$, which gives the desired estimate (5.18) for suitably small $E_{0}+\|\tilde{g}\|$.

Step 4. Finally, we show the decay estimate (5.2). Let $1 / p^{\prime}=1 / p-1 / n$ for $2 \leq p \leq q$. Then we see that $2^{*} \leq p^{\prime} \leq q^{*}$. We have from (4.3c) that $-\Delta\left(\partial_{x} \phi\right)=-\partial_{x}(w-z)$. Therefore, using (2.3) and (5.1), we obtain

$$
\left\|\partial_{x}^{2} \phi(t)\right\|_{L^{p^{\prime}}} \leq C\left\|\partial_{x}(w-z)(t)\right\|_{L^{p}} \leq C E_{0}\left(1+t^{-1 / 2}\right)(1+t)^{-\gamma}=C E_{0}\left(1+t^{-1 / 2}\right)(1+t)^{-\gamma^{\prime}+1 / 2},
$$

where $\gamma=(n / 2)(1 / 2-1 / p)$ and $\gamma^{\prime}=(n / 2)\left(1 / 2-1 / p^{\prime}\right)$; here we have used the relation $\gamma=\gamma^{\prime}-1 / 2$. This shows (5.2) and therefore the proof of Theorem 5.1 is complete.

When $p \geq n$, we have the following $L^{p}$ decay estimate in a weaker sense for the derivatives of solutions.

Theorem $5.2\left(L^{p}\right.$ decay estimates for $\left.p \geq n\right)$. Let $n \geq 3$ and $n \leq q<\infty$. Let $(w, z, \phi)$ be the global solution to the problem (4.3), (4.4) which is constructed in Theorem 4.1 for the initial data $\left(w_{0}, z_{0}\right) \in L^{2} \cap L^{q}$. If $E_{0}+\|\tilde{g}\|$ in Theorem 4.1 is suitably small, then we have the decay estimate (5.1) for each $p$ with $2 \leq p<n$. Moreover, for any small $\varepsilon>0$, we have

$$
\left\|\partial_{x}(w, z)(t)\right\|_{L^{p}} \leq C_{\varepsilon} E_{0}\left(1+t^{-1 / 2}\right)(1+t)^{-\gamma_{n}+\varepsilon}
$$

for each $p$ with $n \leq p \leq q$, where $\gamma_{n}=(n / 2)(1 / 2-1 / n)$, and $C_{\varepsilon}$ is a constant depending on $\varepsilon$. Also we have the decay estimate (5.2) for $\partial_{x}^{2} \phi$ for each $p^{\prime}$ with $2^{*} \leq p^{\prime}<\infty$, where $1 / 2^{*}=1 / 2-1 / n$.

As a simple corollary of Theorems 4.5 and 5.2 , we have the following $L^{\infty}$ decay estimate for $(w, z)$.

Corollary $5.3\left(L^{\infty}\right.$ decay estimate for $\left.(w, z)\right)$. Assume the same conditions as in Theorem 5.2 for $n<q<\infty$. Then, for any small $\varepsilon>0$, we have

$$
\begin{aligned}
& \|(w, z)(t)\|_{L^{\infty}} \leq C_{\varepsilon} E_{0}\left(1+t^{-n / 2 q}\right)(1+t)^{-\gamma_{n}+\varepsilon}, \\
& \left\|\partial_{x}^{2} \phi(t)\right\|_{L^{\infty}} \leq C_{\varepsilon} E_{0}\left(1+t^{-n / 2 q}\right)(1+t)^{-\gamma_{n}+\varepsilon},
\end{aligned}
$$

where $\gamma_{n}=(n / 2)(1 / 2-1 / n)$, and $C_{\varepsilon}$ is a constant depending on $\varepsilon$.

The $L^{\infty}$ decay estimate for $(w, z)$ in $(5.23)$ is easily obtained by using the decay estimates $(4.32)$ and $(5.22)$ for $p=q$ together with the Gagliardo-Nirenberg inequality (2.5) in the form $\|u\|_{L^{\infty}} \leq C\left\|\partial_{x} u\right\|_{L^{q}}^{\theta}\|u\|_{L^{q}}^{1-\theta}$, where $\theta=n / q$ and $q>n$. To show the $L^{\infty}$ decay estimate for $\partial_{x}^{2} \phi$, we recall the estimate (4.35). Also, we have from (4.3c) and (5.22) that $\left\|\Delta \partial_{x} \phi(t)\right\|_{L^{p}} \leq C_{\varepsilon} E_{0}\left(1+t^{-1 / 2}\right)(1+t)^{-\gamma_{n}+\varepsilon}$. Therefore, applying (2.4), we get

$$
\left\|\partial_{x}^{3} \phi(t)\right\|_{L^{p}} \leq C_{\varepsilon} E_{0}\left(1+t^{-1 / 2}\right)(1+t)^{-\gamma_{n}+\varepsilon}
$$

where $n<p \leq q$. Now the desired $L^{\infty}$ decay estimate for $\partial_{x}^{2} \phi$ in (5.23) follows from the same GagliardoNirenberg inequality. 
Proof of Theorem 5.2. Let $n \leq p \leq q$. It suffices to prove the decay estimate (5.22). We use the time weighted $L^{p}$ energy inequality (5.8) for $p \geq n$. For the second term on the right hand side of (5.8), using (4.32) instead of (4.21), we can modify (5.10) as

$$
C \int_{0}^{t} \tau^{\beta p-1}\left\|w_{i}(\tau)\right\|_{L^{p}}^{p} \mathrm{~d} \tau \leq \delta \int_{0}^{t} \tau^{\beta p}\left\|\nabla\left(\left|w_{i}\right|^{p / 2}\right)(\tau)\right\|_{L^{2}}^{2} \mathrm{~d} \tau+C_{\varepsilon, \delta} E_{0}^{p} t^{(\beta-1 / 2) p}(1+t)^{-\left(\gamma_{n}-\varepsilon\right) p}
$$

for $\delta>0$ and $\beta>\gamma_{n}+1 / 2$, where $\gamma_{n}=(n / 2)(1 / 2-1 / n)$, and $C_{\varepsilon, \delta}$ is a constant depending on $(\varepsilon, \delta)$. On the other hand, we see that the estimates (5.11) for $I_{1}^{\prime}$ and (5.16) for $J_{1}^{\prime}$ are valid even for $p \geq n$. Also, using (4.42) instead of (4.41), we can modify (5.12) for $I_{2}^{\prime}$ as

$$
\int_{0}^{t} \tau^{\beta p} I_{2}^{\prime}(\tau) \mathrm{d} \tau \leq C\|\tilde{g}\| \dot{D}_{p, \beta}(t)^{p}+C_{\varepsilon}\|\tilde{g}\| E_{0}^{p} t^{\beta p}(1+t)^{-\left(\gamma_{n}-\varepsilon\right) p}
$$

for $\beta>\gamma_{n}$.

To estimate the term $I_{3}^{\prime}$ for $p \geq n$, we put $\rho=n /(1+2 \varepsilon)$ for small $\varepsilon>0$ and determine $s$ by the relation $1 / s+(1-2 / p) / 2^{*}+1 / 2+1 / \rho^{*}=1$ in (4.38). Then, applying the Hölder inequality with this relation, we can modify (5.13) as

$$
I_{3}^{\prime} \leq C_{\varepsilon}\|\tilde{g}\|\left\|\nabla\left(\left|\partial_{x} w\right|^{p / 2}\right)\right\|_{L^{2}}^{2(1-1 / p)}\|w-z\|_{L^{\rho}} .
$$

Consequently, we can compute similarly as in (4.39) and obtain

$$
\begin{aligned}
\int_{0}^{t} \tau^{\beta p} I_{3}^{\prime}(\tau) \mathrm{d} \tau & \leq C_{\varepsilon}\|\tilde{g}\| \tilde{E}_{\rho, \beta}(t)^{1-\rho / p} \int_{0}^{t} \tau^{\beta p(1-1 / p)+\beta \rho / p}\left\|\nabla\left(\left|\partial_{x} w\right|^{p / 2}\right)\right\|_{L^{2}}^{2(1-1 / p)}\|w-z\|_{L^{\rho}}^{\rho / p} \mathrm{~d} \tau \\
& \leq C_{\varepsilon}\|\tilde{g}\| \tilde{E}_{\rho, \beta}(t)^{1-\rho / p} \dot{D}_{p, \beta}(t)^{p-1} \tilde{D}_{\rho, \beta}(t)^{\rho / p} \\
& \leq C\|\tilde{g}\| \dot{D}_{p, \beta}(t)^{p}+C_{\varepsilon}\|\tilde{g}\| E_{0}^{p} t^{\beta p}(1+t)^{-\left(\gamma_{n}-\varepsilon\right) p}
\end{aligned}
$$

for $\beta>\gamma_{n}$, where we used (4.42). The term $I_{4}^{\prime}$ is estimated similarly. In fact, using $\rho$ and $s$ in (4.38), we can modify (5.14) as

$$
I_{4}^{\prime} \leq C_{\varepsilon}\|\tilde{g}\|\left\|\nabla\left(\left|\partial_{x} w\right|^{p / 2}\right)\right\|_{L^{2}}^{2(1-1 / p)}\left\|\partial_{x}(w-z)\right\|_{L^{\rho}} .
$$

Consequently, similarly as in (4.39), we obtain

$$
\begin{aligned}
\int_{0}^{t} \tau^{\beta p} I_{4}^{\prime}(\tau) \mathrm{d} \tau & \leq C_{\varepsilon}\|\tilde{g}\| \dot{E}_{\rho, \beta}(t)^{1-\rho / p} \int_{0}^{t} \tau^{\beta p(1-1 / p)+\beta \rho / p}\left\|\nabla\left(\left|\partial_{x} w\right|^{p / 2}\right)\right\|_{L^{2}}^{2(1-1 / p)}\left\|\partial_{x}(w-z)\right\|_{L^{\rho}}^{\rho / p} \mathrm{~d} \tau \\
& \leq C_{\varepsilon}\|\tilde{g}\| \dot{E}_{\rho, \beta}(t)^{1-\rho / p} \dot{D}_{p, \beta}(t)^{p-1} \dot{D}_{\rho, \beta}(t)^{\rho / p} \\
& \leq C\|\tilde{g}\| \dot{D}_{p, \beta}(t)^{p}+C_{\varepsilon}\|\tilde{g}\| E_{0}^{p} t^{\beta p}(1+t)^{-\left(\gamma_{n}-\varepsilon\right) p}
\end{aligned}
$$

for $\beta>\gamma_{n}$, where we used (5.9) for $p=\rho$. To estimate $J_{2}^{\prime}$ for $p \geq n$, we again use $\rho$ and $s$ in (4.38). Then we can modify (5.17) as

$$
J_{2}^{\prime} \leq C_{\varepsilon}\|w\|_{L^{s}}\left\|\nabla\left(\left|\partial_{x} w\right|^{p / 2}\right)\right\|_{L^{2}}^{2(1-1 / p)}\left\|\partial_{x}(w-z)\right\|_{L^{\rho}},
$$

which is similar to (5.25). Therefore, using the same computation as in (5.26), we have

$$
\begin{aligned}
\int_{0}^{t} \tau^{\beta p} J_{2}^{\prime}(\tau) \mathrm{d} \tau & \leq C_{\varepsilon} E_{s}(t) \dot{E}_{\rho, \beta}(t)^{1-\rho / p} \int_{0}^{t} \tau^{\beta p(1-1 / p)+\beta \rho / p}\left\|\nabla\left(\left|\partial_{x} w\right|^{p / 2}\right)\right\|_{L^{2}}^{2(1-1 / p)}\left\|\partial_{x}(w-z)\right\|_{L^{\rho}}^{\rho / p} \mathrm{~d} \tau \\
& \leq C_{\varepsilon} E_{s}(t) \dot{E}_{\rho, \beta}(t)^{1-\rho / p} \dot{D}_{p, \beta}(t)^{p-1} \dot{D}_{\rho, \beta}(t)^{\rho / p} \\
& \leq C E_{0} \dot{D}_{p, \beta}(t)^{p}+C_{\varepsilon} E_{0}^{p+1} t^{\beta p}(1+t)^{-\left(\gamma_{n}-\varepsilon\right) p}
\end{aligned}
$$

for $\beta>\gamma_{n}$, where we used (4.6) and (5.9) for $p=\rho$. 
Substituting all these estimates in (5.8) and taking $\delta>0$ in (5.24) suitably small, we arrive at the inequality

$$
\begin{aligned}
& t^{\beta p}\left\|\partial_{x} w(t)\right\|_{L^{p}}^{p}+\int_{0}^{t} \tau^{\beta p}\left\|\nabla\left(\left|\partial_{x} w\right|^{p / 2}\right)(\tau)\right\|_{L^{2}}^{2} \mathrm{~d} \tau+\int_{0}^{t} \tau^{\beta p} \int_{\mathbb{R}^{n}}\left|\partial_{x} w\right|^{p-2} \partial_{x}(w-z) \mathrm{d} x \mathrm{~d} \tau \\
& \leq C_{\varepsilon}\left(1+E_{0}+\|\tilde{g}\|\right) E_{0}^{p} t^{\beta p}\left(1+t^{-p / 2}\right)(1+t)^{-\left(\gamma_{n}-\varepsilon\right) p}+C\left(E_{0}+\|\tilde{g}\|\right)\left(\dot{E}_{p, \beta}+\dot{D}_{p, \beta}\right)(t)^{p}
\end{aligned}
$$

for $\beta>\gamma_{n}+1 / 2$, where $\gamma_{n}=(n / 2)(1 / 2-1 / n)$. Combining this estimate with the corresponding energy inequality for $\partial_{x} z$, we obtain

$$
\left(\dot{E}_{p, \beta}+\dot{D}_{p, \beta}\right)(t)^{p} \leq C_{\varepsilon}\left(1+E_{0}+\|\tilde{g}\|\right) E_{0}^{p} t^{\beta p}\left(1+t^{-p / 2}\right)(1+t)^{-\left(\gamma_{n}-\varepsilon\right) p}+C\left(E_{0}+\|\tilde{g}\|\right)\left(\dot{E}_{p, \beta}+\dot{D}_{p, \beta}\right)(t)^{p},
$$

where $n \leq p \leq q, \beta>\gamma_{n}+1 / 2$ and $\gamma_{n}=(n / 2)(1 / 2-1 / n)$. This gives $\left(\dot{E}_{p, \beta}+\dot{D}_{p, \beta}\right)(t) \leq C_{\varepsilon} E_{0} t^{\beta}\left(1+t^{-1 / 2}\right)(1+$ $t)^{-\gamma_{n}+\varepsilon}$, provided that $E_{0}+\|\tilde{g}\|$ is suitably small. Thus we get the desired decay estimate (5.22). This completes the proof of Theorem 5.2.

Acknowledgements. The authors are deeply grateful to Professors Takayuki Kobayashi and Takayuki Kubo who gave the information on the elliptic estimate in Lemma 2.2. This work was partially supported by Grant-in-Aid for Scientific Research (A) No. 22244009.

\section{REFERENCES}

[1] P. Biler and J. Dolbeault, Long time behavior of solutions to Nernst-Planck and Debye-Hückel drift-diffusion system. Ann. Henri Poincaré 1 (2000) 461-472.

[2] P. Biler and N. Nadzieja, Existence and nonexistence of solutions for a model of gravitational interactions of particles I. Colloq. Math. 66 (1994) 319-334.

[3] P. Biler and N. Nadzieja, A singular problem in electrolytes theory. Math. Methods Appl. Sci. 20 (1997) 767-782.

[4] P. Biler, G. Karch, P. Laurençot and T. Nadzieja, The $8 \pi$-problem for radially symmetric solutions of a chemotaxis model in the plane. Math. Methods Appl. Sci. 29 (2006) 1563-1583.

[5] S. Childress and J.K. Percus, Nonlinear aspects of chemotaxis, Math. Biosci. 56 (1981) $217-237$.

[6] R. Farwig and H. Sohr, Weighted $L^{q}$-theory for the Stokes resolvent in exterior domains. J. Math. Soc. Jpn 49 (1997) $251-288$.

[7] M. Escobedo and E. Zuazua, Large time behavior for convection-diffusion equations in $\mathbb{R}^{N}$. J. Funct. Anal. 100 (1991) 119-161.

[8] S. Kawashima, S. Nishibata and M. Nishikawa, $L^{p}$ energy method for multi-dimensional viscous conservation laws and application to the stability of planar waves. J. Hyperbolic Differ. Equ. 1 (2004) 581-603.

[9] E.F. Keller and L.A. Segel, Initiation of slime mold aggregation viewed as an instability, J. Theor. Biol. 26 (1970) $399-415$.

[10] R. Kobayashi and S. Kawashima, Decay estimates and large time behavior of solutions to the drift-diffusion system. Funkcial. Ekvac. 51 (2008) 371-394.

[11] R. Kobayashi, M. Kurokiba and S. Kawashima, Stationary solutions to the drift-diffusion model in the whole space. Math. Methods Appl. Sci. 32 (2009) 640-652.

[12] M. Kurokiba and T. Ogawa, $L^{p}$ wellposedness for the drift-diffusion system arising from the semiconductor device simulation. J. Math. Anal. Appl. 342 (2008) 1052-1067.

[13] D.S. Kurtz and R.L. Wheeden, Results on weighted norm inequalities for multipliers, Trans. Amer. Math. Soc. 255 (1979) 343-362.

[14] M.S. Mock, Asymptotic behavior of solutions of transport equations for semiconductor devices, J. Math. Anal. Appl. 49 (1975) 215-225.

[15] T. Nagai, Global existence and decay estimates of solutions to a parabolic-elliptic system of drift-diffusion type in $\mathbb{R}^{2}$. Differential Integral Equations 24 (2011) 29-68.

[16] L. Nirenberg, On elliptic partial differential equations. Ann. Scuola Norm. Sup. Pisa 13 (1959) 115-162.

[17] A. Raczyński, Weak- $L^{p}$ solutions for a model of self-gravitating particles with an external potential. Stud. Math. 179 (2007) 199-216.

[18] D.R. Smart, Fixed Point Theorems. Cambridge University Press, New York (1974).

[19] E.M. Stein, Singular Integrals and Differentiability Properties of Functions. Princeton University Press, Princeton (1970).

[20] G. Wolansky, On steady distributions of self-attracting clusters under friction and fluctuations, Arch. Rational Mech. Anal. 119 (1992) 355-391.

[21] W.P. Ziemer, Weakly Differentiable Functions. Springer-Verlag, New York (1989). 